\title{
Plactic relations for $r$-domino tableaux
}

\author{
Müge Taşkın * \\ Department of Mathematics \\ Bog̃aziçi University, İstanbul, Türkiye \\ muge.taskin@boun.edu.tr
}

Submitted: Feb 8, 2011; Accepted: Jan 27, 2012; Published: Feb 7, 2012

Mathematics Subject Classification: 05E10

\begin{abstract}
The work of C. Bonnafé, M.Geck, L. Iancu and T. Lam [3] shows through two conjectures that $r$-domino tableaux have an important role in Kazhdan-Lusztig theory of type $B$ with unequal parameters. In this paper we provide plactic relations on signed permutations which determine whether two given signed permutations have the same insertion $r$-domino tableaux in Garfinkle's algorithm [4]. Moreover, we show that a particular extension of these relations can describe Garfinkle's equivalence relation [4] on $r$-domino tableaux which is given through the notion of open cycles. With these results we enunciate the conjectures of [3] and provide necessary tools for their proofs.
\end{abstract}

\section{Introduction}

Let $W$ be a finite Coxeter group and let $L: W \mapsto \mathbb{Z}_{\geq 0}$ be a weight function such that

$$
L(u w)=L(u)+L(w) \text { if and only if } l(u w)=l(u)+l(w)
$$

where $l: W \mapsto \mathbb{Z}_{\geq 0}$ is the usual length function on $W$. As it is described by Lusztig in [14] every weight function determines an Iwahori-Hecke algebra and three preorders on $W$ whose equivalence classes are called left, right and two-sided cells. The importance of these cells lies in the fact that they carry representations of $W$ and its corresponding Iwahori-Hecke algebra $\mathcal{H}$. Furthermore they have an important role in the representation theory of reductive algebraic groups over finite or $p$-adic fields [14] and in the study of rational Cherednik algebras [8] and the Calogero-Moser spaces [9].

The case $L=l$ is in fact first introduced by Kazhdan and Lusztig in [11] as a purely combinatorial tool for the theory of primitive ideals in the universal enveloping algebras of

\footnotetext{
${ }^{*}$ This research was partially supported by the Fields Institute and York University, Toronto, ON, CA
} 
semisimple complex Lie algebras. In this case the combinatorial characterizations of cells are well known, where Knuth (or plactic) relations appear as the mediating tool. Namely, when $W$ is type $A$ then each right (left) cell corresponds to the plactic (respectively coplactic) class of some standard Young tableau, whereas each two-sided cell consists of those permutations which lie in the plactic classes of tableaux of the same shape. This characterizations depend on Joseph's classification of primitive ideals in type A, where Knuth (plactic) relations play a crucial role.

In the types $\mathrm{B}, \mathrm{C}$ and $\mathrm{D}$, on the other hand the emerging combinatorial objects are standard domino tableaux. The connection is first revealed in the work of Barbash and Vogan [1] where they provide necessary conditions for the characterizations of primitive ideals through an algorithm which uses the palindrome representations of signed permutations in order to assign to every signed permutation $\alpha$ a pair of same shape standard $r$-domino tableaux $\left(P^{r}(\alpha), Q^{r}(\alpha)\right)$ bijectively, for $r=0$ or $r=1$. Meanwhile, an analog of Knuth relations provided by Joseph in [10] established the sufficient conditions. On the other hand Garfinkle [4, 5,6] finalized the classification problem for these types by showing through her two algorithms on domino tableaux that these two sets of relations are in fact equivalent. Her first algorithm assigns any signed permutation to a pair of same shape standard $r$-domino tableaux for $r$ equal to 0 or 1 and the second defines an equivalence relation between domino tableaux through the notion of open cycles. We remark that the extension of Garfinkle and Barbash-Vogan algorithm for larger $r$ is given in [13] and [3] respectively.

The case $L \neq l$ is also known as unequal parameter Kazhdan-Lusztig theory and it appears for the types $B_{n}, I_{2}(n)$ and $F_{4}$, where the classification problem for the latter two can be dealt with computational methods, see [7]. For type $B_{n}$, the weight function is determined by two integers $a, b>0$ such that

$$
L\left(s_{i}\right)= \begin{cases}a & \text { if } 1 \leq i \leq n-1 \\ b & \text { if } i=0\end{cases}
$$

where $s_{0}$ is the transposition $(-1,1)$ and $\left\{s_{i}=(i, i+1) \mid 1 \leq i \leq n-1\right\}$ are the type $A$ generators of $B_{n}$. Recently, the role of $r$-domino tableaux in this theory is revealed in the work of Bonnafé, Geck, Iancu, and Lam [3] through two main conjectures:

- Conjecture A: If $r a<b<(r+1) a$ for some $r \geq 0$ then two signed permutations lie in the same Kazhdan-Lusztig right (left) cell if and only if their insertion (recording) $r$-domino tableau are the same.

- Conjecture B: If $b=r a$ for some $r \geq 1$ then two signed permutations lie in the same Kazhdan-Lusztig right (left) cell if and only if their insertion (recording) $r-1$ domino tableau are equivalent through the notion of open cycles.

In order to establish the proofs of these conjecture one definitely needs the plactic relations between signed permutations which determines when the insertion $r$-domino tableaux of two signed permutations are the same or equivalent through the notion of open cycles. Our aim here is to fill this gap. 
This paper is organized as follows: The descriptions of Barbash-Vogan and Garfinkle's algorithms can be found in Section 2 together with some lemmas which are essential in the following section. In Section 3 the definition of plactic relations are given and they are shown to be necessary and sufficient for describing plactic classes of $r$-domino tableaux.

Remark. Recently T. Pietraho [18] has found another set of generators which can be shown to be equivalent to $\mathrm{D}_{1}^{r}, \mathrm{D}_{2}^{r}, \mathrm{D}_{3}^{r}$ and $\mathrm{D}_{3}^{r-1}$ given in the Definition 13 . On the other hand these relations describes a larger set, namely the set of all permutations whose insertion $r$-domino tableaux are equivalent through the notion of open cycles. Finally, by using his results and an earlier version of the present work, C. Bonnafé provides a partial result towards the previous conjectures [2].

\section{Acknowledgments}

The author is grateful to the referee for careful reading and a number of helpful suggestions for improvement in the article. The author also thanks to Nantel Bergeron, Victor Reiner and Huilan Li for helpful discussions and comments.

\section{Related background}

A sequence $\lambda=\left(\lambda_{1}, \ldots, \lambda_{k}\right)$ is a partition of $n$, denoted by $\lambda \vdash n$, if $\sum_{i=1}^{k} \lambda_{i}=n$ and $\lambda_{i} \geq$ $\lambda_{i+1}>0$ where its Ferrers diagram consists of left justified arrows of boxes such that the $i$-th row has $\lambda_{i}$ boxes. For example

$$
\lambda=(2,2,1)=\square
$$

A partition $\lambda=\left(\lambda_{1}, \ldots, \lambda_{k}\right)$ can be also seen as a set of integer pairs $(i, j)$ such that $1 \leq i \leq k$ and $1 \leq j \leq \lambda_{i}$. Therefore for two partitions $\lambda$ and $\mu$, we can define usual set operations such as $\lambda \cup \mu, \lambda \cap \mu, \lambda \subset \mu, \lambda-\mu$, but the resulting sets do not necessarily correspond to any partitions.

Definition 1 For two partitions $\lambda$ and $\mu$ satisfying $\mu \subset \lambda$ we define $\lambda / \mu=\lambda-\mu$ to be the skew partition determined by $\lambda$ and $\mu$.

Definition 2 Let $\gamma$ and $\gamma^{\prime}$ be two skew shapes.

1. If $\gamma \cap \gamma^{\prime}=\emptyset$ and $\gamma \cup \gamma^{\prime}$ also corresponds a skew shape then we define $\gamma \oplus \gamma^{\prime}=\gamma \cup \gamma^{\prime}$.

2. If $\gamma^{\prime} \subset \gamma$ and $\gamma-\gamma^{\prime}$ also corresponds a skew shape then we define $\gamma \ominus \gamma^{\prime}=\gamma-\gamma^{\prime}$.

Definition 3 Let $\lambda$ be a partition and $(i, j) \in \lambda$.

1. If $(i, j) \in \lambda$ and $\lambda \ominus(i, j)$ is also a partition then $(i, j)$ is called a corner of $\lambda$. 
2. If $(i, j) \notin \lambda$ and $\lambda \oplus(i, j)$ is also a partition then $(i, j)$ is called an empty corner of $\lambda$.

Definition 4 A skew tableau $T$ of shape $\lambda / \mu$ is obtained by labeling the cells of $\lambda / \mu$ with non repeating, totally ordered letters such that the letters increase from left to right and from top to bottom. If $\mu=\emptyset$ then $T$ is called a Young tableau. We denote by

$$
\text { label }(T) \text { and } \operatorname{shape}(T)
$$

respectively, the set of letters labeling each box of $T$ and the partition underlying $T$. For a set $\mathcal{A}$ of letters given with a totally ordering, we denote by $S Y T_{\mathcal{A}}$ the set of Young tableaux labeled with $\mathcal{A}$. If $\mathcal{A}=\{1,2, \ldots, n\}$ then $T$ is called a standard skew or standard Young tableau according to the shape of T. Moreover the set of standard Young tableaux are denoted by $S Y T_{n}$.

Let $S_{\mathcal{A}}\left(S_{n}\right)$ denote the symmetric group on the totaly ordered set $\mathcal{A}$ (respectively on $\{1,2, \ldots, n\})$. When the size of $\mathcal{A}$ is $n$ we have an order preserving bijection between $\mathcal{A}$ and $\{1,2, \ldots, n\}$ and this yields two more bijections between $S_{\mathcal{A}}$ and $S_{n}$ as well as $S Y T_{\mathcal{A}}$ and $S Y T_{n}$. Therefore the following discussions and results can be generalized to any finite totally ordered set $\mathcal{A}$.

There is an important connection, between Young tableaux $S Y T_{n}$ and the symmetric group $S_{n}$, known as the Robinson-Schensted correspondence (RSK), which was realized by Robinson and Schensted independently. In this correspondence, every permutation $w \in$ $S_{n}$ is assigned bijectively to a pair of same shape tableaux $(P(w), Q(w))$ in $S Y T_{n} \times S Y T_{n}$ through insertion and recording algorithms. Let us explain these algorithms briefly. We denote by $\left(P_{i-1}, Q_{i-1}\right)$ the tableaux obtained by insertion and recording algorithms on the first $i-1$ indices of $w=w_{1} \ldots w_{n}$. In order to get $P_{i}$ we proceed as follows: if $w_{i}$ is greater then the last number on the first row of $P_{i-1}$, then $w_{i}$ is concatenated to the first row of $P_{i-1}$ from the right, otherwise $w_{i}$ replaces the smallest number, say $a$, among all numbers in the first row which are greater then $w_{i}$ and the insertion algorithm continues with the insertion of $a$ to the next row. Observe that after finitely many steps the insertion algorithm terminates with a new appearing cell on some row of $P_{i-1}$. The resulting tableau is then $P_{i}$ and the recording tableau $Q_{i}$ is found by filling this new cell in $Q_{i-1}$ with the number $i$. We illustrate these algorithms with the following example.

Example 1 Let $w=52413 \in S_{5}$. Then,

$$
\begin{aligned}
& P_{1}=5, \quad P_{2}=\begin{array}{l}
2 \\
5
\end{array}, \quad P_{3}=\begin{array}{l}
4 \\
5
\end{array} \quad, \quad P_{4}=\begin{array}{l}
1 \\
2 \\
5
\end{array} \quad, \quad P_{5}=\begin{array}{ll}
1 & 3 \\
2 & 4 \\
5
\end{array} \quad=P(w) \\
& Q_{1}=1, \quad Q_{2}=\begin{array}{l}
1 \\
2
\end{array}, \quad Q_{3}=\begin{array}{ll}
1 & 3 \\
2
\end{array}, \quad Q_{4}=\begin{array}{l}
1 \\
2 \\
4
\end{array} \quad, \quad Q_{5}=\begin{array}{ll}
1 & 3 \\
2 & 5 \\
4
\end{array} \quad=Q(w)
\end{aligned}
$$

The following result of Schützenberger [20] reveals an important property of the RSK. 
Theorem 2.1 If $w \in S_{n}$, then $P\left(w^{-1}\right)=Q(w)$ and $Q\left(w^{-1}\right)=P(w)$.

There are two equivalence relations and a related result given by Knuth [12] which are fundamental in the combinatorics of tableaux. In the following we provide them in a more general setting:

Definition 5 Let $\mathcal{A}$ be a totally ordered set of letters and $u=u_{1} \ldots u_{n} \in S_{\mathcal{A}}$. If either $u_{i}<u_{i+2}<u_{i+1}$ or $u_{i}<u_{i-1}<u_{i+1}$ for some $i$ then

$$
u=u_{1} \ldots u_{i-1}\left(u_{i} u_{i+1}\right) u_{i+2} \ldots u_{n} \sim u_{1} \ldots u_{i-1}\left(u_{i+1} u_{i}\right) u_{i+2} \ldots u_{n}=u^{\prime} .
$$

We say $u, w \in S_{\mathcal{A}}$ are Knuth equivalent, $u \stackrel{K}{\sim} w$, if $w$ can be obtained from $u$ by applying a sequence of $\sim$ relations. On the other hand if $u^{-1} \stackrel{K}{\sim} w^{-1}$ then $u$ and $w$ are called dual Knuth equivalent, $u \stackrel{K^{*}}{\sim} w$.

Theorem 2.2 (Knuth [12]) Let $u, w \in S_{\mathcal{A}}$. Then
i) $u \stackrel{K}{\cong} w \Longleftrightarrow P(u)=P(w)$
ii) $u \stackrel{K^{*}}{\cong} w \Longleftrightarrow Q(u)=Q(w)$.

We next illustrate the forward and backward slides of Schützenberger's jeu de taquin [21] without the definition. We remark that jeu de taquin slides can be used to give alternative descriptions of both the Robinson-Schensted algorithm and Knuth relations. The following theorem provided by Schützenberger in [21] reveal this connection.

Example 2 Below we illustrate a forward slide on the tableau $S$ through cell $c_{12}$ and backward slide on the tableau $T$ through cell $c_{32}$.
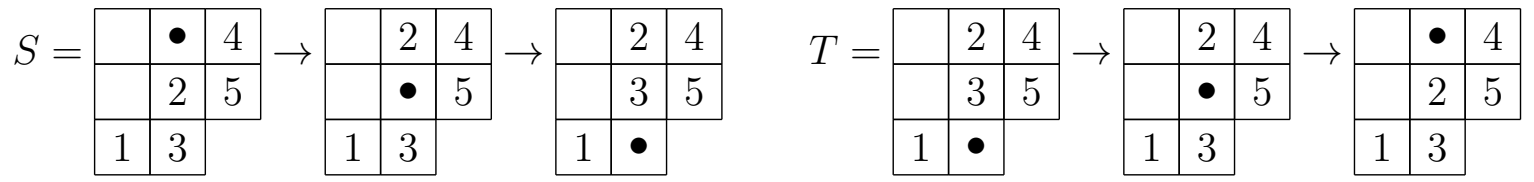

Theorem 2.3 If $P$ is a skew tableau that is brought to a Young tableau $P^{\prime}$ by slides, then $P^{\prime}$ is unique. In fact, $P^{\prime}$ is the insertion tableau for the row word of $P$.

Definition 6 The set of two adjacent cells $A=\{(i, j),(i, j+1)\}$ (or $A=\{(i, j),(i+1, j)\})$ is called a horizontal (or respectively vertical) domino cell. Now

$$
\min (A) \text { and } \max (A)
$$

denotes the minimum and respectively maximum cell of $A$ in the lexicographic order. Let $A=\left\{\left(r_{1}, c_{1}\right),\left(r_{1}^{\prime}, c_{1}^{\prime}\right)\right\}$ and $B=\left\{\left(r_{2}, c_{2}\right),\left(r_{2}^{\prime}, c_{2}^{\prime}\right)\right\}$ be two domino cells in $T$ where $\max (A)=\left(r_{1}^{\prime}, c_{1}^{\prime}\right)$ and $\min (B)=\left(r_{2}, c_{2}\right)$. Then we say 
i) $B$ lies below $A$ if $\min (B)$ lies below $\max (A)$, equivalently $r_{2}>r_{1}^{\prime}$.

ii) $B$ lies to the right of $A$ if $\min (B)$ lies to the right of $\max (A)$, equivalently $c_{2}>c_{1}^{\prime}$.

Let $\lambda$ be a partition and $A$ be a domino cell. If $\lambda \oplus A$ is a partition then $A$ is called an empty domino corner of $\lambda$ whereas if $\lambda \ominus A$ is also a partition then $A$ is called a domino corner of $\lambda$. Clearly, if a partition has no domino corner then it must be a $r$-staircase shape $(r, \ldots, 2,1)$ for some $r>0$. On the other hand it is easy to see that any partition $\lambda$ can be reduced uniquely to a $r$-staircase shape $(r, \ldots, 2,1)$ for some $r \geq 0$, by subsequent removal of existing domino corners one at a time. In this case we say $\lambda$ has a 2 -core equivalent to $(r, \ldots, 2,1)$. For $r \geq 0$ we denote by $P(2 n, r)$ the set of all such partitions of size $2 n+r(r+1) / 2$.

Definition 7 By a labeling of domino cell $A$ we mean a pair of positive numbers $\left(a, a^{\prime}\right)$ which label the boxes of $A$ such that $a \leq a^{\prime}$ and a labels $\min (A)$ and $a^{\prime}$ labels $\max (A)$. If the label of $A$ is $(a, a)$ then we say $A$ is double labeled by $a$. When we want to indicate the domino cell $A$ with its labeling, we use the notation

$$
\left[A,\left(a, a^{\prime}\right)\right]
$$

so that shape $\left(\left[A,\left(a, a^{\prime}\right)\right]\right)=A$ and $\operatorname{label}\left(\left[A,\left(a, a^{\prime}\right)\right]\right)=\left(a, a^{\prime}\right)$.

A r-domino tableau $T$ of shape $\lambda \in P(2 n, r)$ is obtained by tiling the skew partition $\lambda /(r, \ldots, 2,1)$ with double labeled horizontal or vertical domino cells $\left\{\left[A_{1},\left(a_{1}, a_{1}\right)\right]\right.$, $\left.\ldots\left[A_{n},\left(a_{n}, a_{n}\right)\right]\right\}$ such that $a_{i}>0$ for all $i=1, \ldots, n, a_{i} \neq a_{j}$ for $i \neq j$ and the labels increase from left to right and from top to bottom. In this case we have

$$
\operatorname{label}(T)=\left\{a_{1}, a_{2}, \ldots, a_{n}\right\} .
$$

A standard $r$-domino tableau $T$ is a $r$-domino tableau which has label $(T)=\{1, \ldots, n\}$. We denote by $S D T^{r}(n)$ the set of all standard $r$-domino tableaux of $n$ dominos.

Definition 8 Let $T$ be a $r$-domino tableau and $\lambda=\operatorname{shape}(T)$. For $A$ is a domino cell in $\lambda$ and $b \in \operatorname{label}(T)$ we define,

1. label $(T, A)$ to be the pair of integers $\left(a, a^{\prime}\right)$ which label the domino cell $A$ in $T$, where $a \leq a^{\prime}$.

2. $\operatorname{Dom}(T, b)=[B,(b, b)]$ if $B$ is a domino cell double labeled by $b$ in $T$.

Example 3 For example the following is a 2-domino tableau in $\operatorname{SDT}^{2}(5)$.

$$
T=\begin{array}{|l|l|l|l|l|}
\hline & & 1 & 1 & 5 \\
\hline & 3 & 4 & 4 & 5 \\
\cline { 1 - 2 } 2 & 3 & \multicolumn{3}{|l}{} \\
\cline { 1 - 2 } 2 & \multicolumn{3}{|c}{} \\
\cline { 1 - 1 }
\end{array}
$$

Here $T$ has two domino corners: $A_{1}=\{(1,5),(2,5)\}$ and $A_{2}=\{(2,4),(2,5)\}$, whereas label $\left(T, A_{1}\right)=(5,5)$ and label $\left(T, A_{2}\right)=(4,5)$. On the other hand $\operatorname{Dom}(T, 5)=\left[A_{1},(5,5)\right]$. 
Definition 9 For two $r$-domino tableau $S$ and $T$ satisfying $S \subset T$ we define $T / S=T-S$ to be the skew $r$-domino tableau determined by $S$ and $T$.

Definition 10 Let $R$ and $R^{\prime}$ be two skew $r$-domino tableaux and let shape $(R)=\gamma$ and $\operatorname{shape}\left(R^{\prime}\right)=\gamma^{\prime}$.

1. If $\gamma \oplus \gamma^{\prime}$ is defined and $R \cup R^{\prime}$ corresponds to a skew $r$-domino tableau as a set then we define $R \oplus R^{\prime}=R \cup R^{\prime}$

2. If $\gamma \ominus \gamma^{\prime}$ is defined and if $R-R^{\prime}$ corresponds to a skew $r$-domino tableau as a set then we define $R \ominus R^{\prime}=R-R^{\prime}$

Definition 11 Let $T$ be a (skew) r-domino tableau and $a \in \operatorname{label}(T)$. Then we define

1. $T_{<a}\left(T_{\leq a}\right)$ to be the $r$-domino tableau obtained by restricting $T$ to its double labeled domino cells whose labels are less than (and equal to) a.

2. $T_{>a}\left(T_{\geq a}\right)$ to be the skew $r$-domino tableau obtained by restricting $T$ to its double labeled domino cells whose labels are greater than (and equal to) a.

\subsection{Garfinkle's algorithm}

Recall that a signed permutation $\alpha \in B_{n}$ is a bijection of $[-n,+n]$ such that $\alpha(-i)=$ $-\alpha(i)$. The usual presentation of $\alpha \in B_{n}$ is denoted as $\alpha=\alpha_{1} \alpha_{2} \ldots \alpha_{n}$ where $\alpha_{i}=\alpha(i)$ for $1 \leq i \leq n$ and $\left\{\left|\alpha_{1}\right|,\left|\alpha_{2}\right| \ldots,\left|\alpha_{n}\right|\right\}=\{1,2, \ldots, n\}$. In the following we set the following representation for all integers:

$$
\bar{a}=\left\{\begin{aligned}
-a & \text { if } a>0 \\
|a| & \text { if } a<0
\end{aligned}\right.
$$

Garfinkle [4, Theorem 1.2.13] provides an algorithm by which any signed permutation $\alpha \in B_{n}$ is assigned bijectively to a pair of same shape standard $r$-domino tableau $\left(P^{r}(\alpha), Q^{r}(\alpha)\right)$ for $r=0,1$, where $P^{r}(\alpha)$ is called the insertion and $Q^{r}(\alpha)$ is called the recording tableau of $\alpha$. Her algorithm is extended by van Leeuwen [13] for larger cores.

In the following we will explain how to insert an integer into a $r$-domino tableau according to Garfinkle's algorithm. Let $T$ be a $r$-domino tableau such that $|a| \notin \operatorname{label}(T)$. We denote by

$$
T^{\downarrow a}
$$

the tableau which is obtained by inserting $a$ into $T$.

Let $a_{0}$ be the largest label in $T$ which is smaller then $|a|$. If $a>0$ then we first concatenate a horizontal domino labeled with $(a, a)$ to the first row of $T_{\leq a_{0}}$ from the right. Otherwise a vertical domino labeled with $(|a|,|a|)$ is concatenated to the first column of $T_{\leq a_{0}}$ from the bottom. Let $I_{0}$ denote the resulting tableau. If the skew tableau $T_{>a_{0}}$ is empty then we have

$$
T^{\downarrow a}=I_{0} .
$$


Otherwise let $a_{1}, a_{2}, \ldots, a_{s}$ be the increasing sequence of the labels in $T_{>a}$. In the following we will find $T^{\downarrow a}$ through a sequence of tableaux $I_{0}, I_{1} \ldots, I_{s}$ where

$$
T^{\downarrow a}=I_{s}=I_{s-1} \leftarrow \operatorname{Dom}\left(T, a_{s}\right)=\ldots=I_{0} \leftarrow \operatorname{Dom}\left(T, a_{1}\right) \leftarrow \ldots \leftarrow \operatorname{Dom}\left(T, a_{s}\right)
$$

and for each $i=1, \ldots, s$,

$$
I_{i}=I_{i-1} \leftarrow \operatorname{Dom}\left(T, a_{i}\right)
$$

is obtained by sliding $\operatorname{Dom}\left(T, a_{i}\right)$ to the tableau $I_{i-1}$ in the following manner: Let

$$
B_{i}=\operatorname{shape}\left(I_{i-1}\right) \cap \operatorname{shape}\left(\operatorname{Dom}\left(T, a_{i}\right)\right)
$$

We first assume that $\operatorname{Dom}\left(T, a_{i}\right)=\left[\{(k, l),(k, l+1)\},\left(a_{i}, a_{i}\right)\right]$ is horizontal. Then we have the following possibilities:

$\left.H_{1}\right) B_{i}=\emptyset$. Then $I_{i}=I_{i-1} \leftarrow \operatorname{Dom}\left(T, a_{i}\right)=I_{i-1} \oplus \operatorname{Dom}\left(T, a_{i}\right)$.

$\left.H_{2}\right) B_{i}=\{(k, l),(k, l+1)\}$. Then in order to obtain $I_{i}=I_{i-1} \leftarrow \operatorname{Dom}\left(T, a_{i}\right)$, a horizontal domino cell double labeled by $a_{i}$ is concatenated to the $(k+1)$-th row of $I_{i-1}$ from the right.

$\left.H_{3}\right) B_{i}=\{(k, l)\}$. Then

$$
I_{i}=I_{i-1} \leftarrow \operatorname{Dom}\left(T, a_{i}\right)=I_{i-1} \oplus\left[\{(k, l+1),(k+1, l+1)\},\left(a_{i}, a_{i}\right)\right] .
$$

Now we assume that $\operatorname{Dom}\left(T, a_{i}\right)=\left[\{(k, l),(k+1, l)\},\left(a_{i}, a_{i}\right)\right]$ is vertical. Then we have the following possibilities for $B_{i}$ :

$\left.V_{1}\right) B_{i}=\emptyset$. Then $I_{i}=I_{i-1} \leftarrow \operatorname{Dom}\left(T, a_{i}\right)=I_{i-1} \oplus \operatorname{Dom}\left(T, a_{i}\right)$.

$\left.V_{2}\right) B_{i}=\{(k, l),(k+1, l)\}$. Then in order to find $I_{i}=I_{i-1} \leftarrow \operatorname{Dom}\left(T, a_{i}\right)$, a vertical domino cell double labeled by $a_{i}$ is concatenated to the $(l+1)$-th column of $I_{i-1}$ from the bottom.

$\left.V_{3}\right) B_{i}=\{(k, l)\}$. Then

$$
I_{i}=I_{i-1} \leftarrow \operatorname{Dom}\left(T, a_{i}\right)=I_{i-1} \oplus\left[\{(k+1, l),(k+1, l+1)\},\left(a_{i}, a_{i}\right)\right] .
$$

Then insertion and recording $r$-domino tableaux for any $\alpha=\alpha_{1} \ldots \alpha_{n}$ is found in the following way: Suppose that $P_{0}$ and $Q_{0}$ are the tableaux of shape $(r, \ldots, 2,1)$ whose cells are all filed with 0 . For $\alpha=\alpha_{1} \ldots \alpha_{n} \in B_{n}$ let $P_{i+1}=P_{i}^{\downarrow \alpha_{i}}$ and let $Q_{i+1}$ be obtained from $Q_{i}$ by filing the newly appearing the domino corner of $P_{i+1}$ with $(i+1, i+1)$ in $Q_{i+1}$. Then one can obtain $P^{r}(\alpha)$ and $Q^{r}(\alpha)$ by erasing all zeros of $P_{n}$ and respectively $Q_{n}$.

The following lemma directly follows from Garfinkle's algorithm and it indicates some of its main features. 
Lemma 2.4 Let $n$ be the largest entry in $T$ and $a$ be an integer satisfying $|a|<n$ and $a \notin \operatorname{label}(T)$. Then

i. $T^{\downarrow \bar{a}}=\left(\left(T^{t}\right)^{\downarrow a}\right)^{t}$ where $T^{t}$ is the transpose of $T$.

ii. $T^{\downarrow a}=\left(T_{<n}\right)^{\downarrow a} \leftarrow \operatorname{Dom}(T, n)$

Example 4 Find $T^{\downarrow \overline{2}}$ for $T=$\begin{tabular}{|l|l|l|l|}
\hline 1 & 1 & 3 & 3 \\
\hline 4 & 5 & 5 \\
\cline { 1 - 2 } 4 & 6 & 6
\end{tabular} , where $T_{<2}=$\begin{tabular}{|l|l|}
\hline 1 & 1 \\
\hline
\end{tabular} .

$$
\begin{aligned}
& I_{0}=\begin{array}{|l|l|}
\hline 1 & 1 \\
\hline 2 & \\
\cline { 1 - 1 } 2 &
\end{array}
\end{aligned}
$$

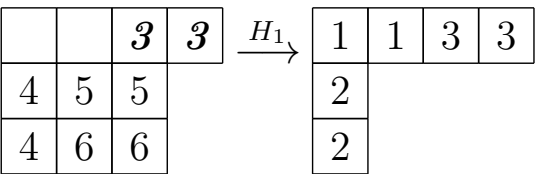

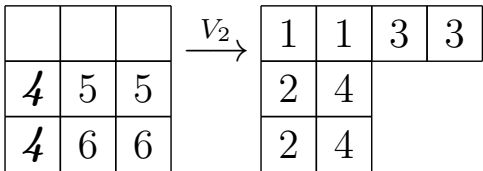

$$
\begin{aligned}
& \stackrel{H_{3}}{\longrightarrow} \begin{array}{|l|l|l|l|}
\hline 1 & 1 & 3 & 3 \\
\cline { 1 - 2 } 2 & 4 & 5 & \\
\cline { 1 - 2 } 2 & 4 & 5 &
\end{array}
\end{aligned}
$$

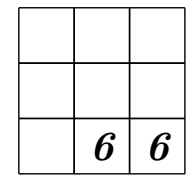

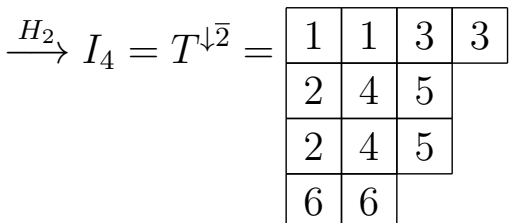$$
\begin{array}{|l|l|l|}
\hline & & \\
\hline & \mathbf{5} & \mathbf{5} \\
\hline & 6 & 6 \\
\hline
\end{array}
$$

We now explain the reverse-insertion of domino corners from $r$-domino tableaux which is the main ingredient of Garfinkle's bijection. Let $T$ be a $r$-domino tableau and $A$ be a domino corner in shape $(T)$. We denote by

$$
T^{\uparrow A} \text { and } \eta\left(T^{\uparrow A}\right)
$$

respectively the tableau which is obtained by the reverse-insertion of $A$, and the number which is bumped out of $T$ as a result of this operation. Clearly, one has

$$
\left(T^{\uparrow A}\right)^{\downarrow \eta\left(T^{\uparrow A}\right)}=T .
$$

Direct use of Garfinkle insertion algorithm gives the following result where the bold letters indicate the domino cell which is pushed back during the reverse insertion algorithm.

Corollary 2.5 Let $T$ be an $r$-domino tableau and $A$ is a domino corner. Further let $A^{\prime}$ be the domino cell which is pushed back by $A$ in the first step of the reverse insertion $T^{\uparrow A}$. Then

i) If $A=\{(i, j),(i, j+1)\}$ and $\operatorname{label}(T, A)=(a, a)$ then $A^{\prime} \subset\{(i-1, k) \mid k \geq j\}$.

ii) If $A=\{(i, j),(i, j+1)\}$ and $\operatorname{label}(T, A)=\left(a^{\prime}, a\right)$ for some $a^{\prime}<a$ then $A^{\prime}=$ $\{(i-1, j),(i, j)\}$.
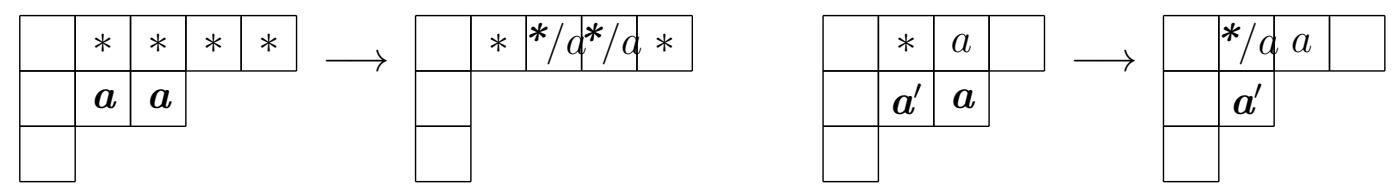
iii) If $A=\{(i, j),(i+1, j)\}$ and $\operatorname{label}(T, A)=(a, a)$ then $A^{\prime} \subset\{(k, j-1) \mid k \geq i\}$.

iv) If $A=\{(i, j),(i+1, j)\}$ and $\operatorname{label}(T, A)=\left(a^{\prime}, a\right)$ for some $a^{\prime}<$ a then $A^{\prime}=$ $\{(i, j-1),(i, j)\}$.
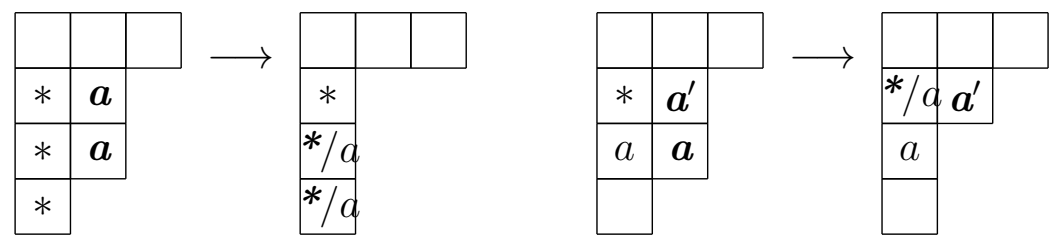

Example 5 Let $T \in S D T^{3}(5)$ and $B=\{(4,2),(5,3)\}$ as given below. Then one can obtain $T^{\uparrow B}$ in the following manner:

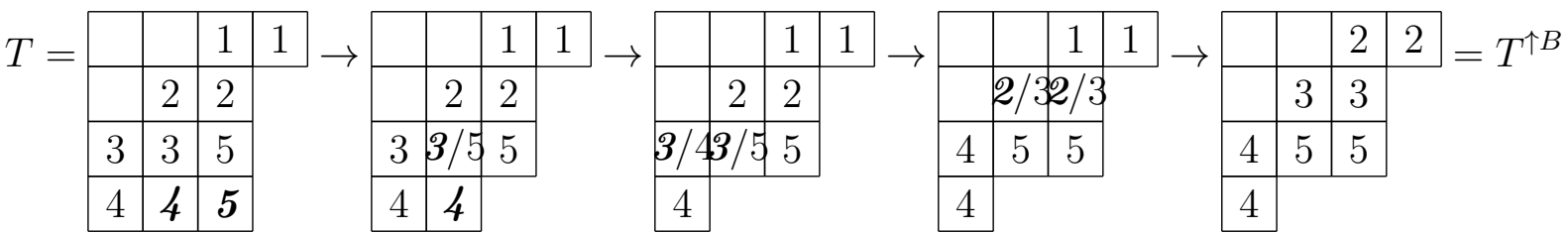

Moreover $\eta\left(T^{\uparrow B}\right)=1$.

\subsection{Barbash and Vogan algorithm.}

We will now explain the algorithm which is provided by Barbash and Vogan in [1] to establish the bijection between signed permutations and standard $r$-domino tableaux for $r=0,1$ where $r=0$ represents type $C$ and $r=1$ represents type $B$ signed permutations. The extension of this algorithm for larger cores is provided in [3]. We also remark that the equivalence of Barbash-Vogan algorithm to Garfinkle's algorithm for $r=0,1$, is due to van Leeuwen [13] .

Recall that for a signed permutation $\alpha=\alpha_{1} \alpha_{2} \ldots \alpha_{n}$ the palindrome representation of $\alpha$ is given by $\alpha^{0}=\bar{\alpha}_{n} \ldots \bar{\alpha}_{2} \bar{\alpha}_{1} \alpha_{1} \alpha_{2} \ldots \alpha_{n}$ where $\bar{\alpha}_{i}=-\alpha_{i}$. We call $\alpha^{0}$ as 0 -core representation of $\alpha$. Clearly 0 -core representation defines an injective map from the set of all signed permutations of size $n$ into $S_{2 n}$.

By following the approach of [3] let us describe how to extend this representation for larger cores. We first identify $\{1,2, \ldots, r(r+1) / 2\}$ with $\left\{0_{1}, 0_{2}, \ldots, 0_{r(r+1) / 2}\right\}$ together with the total ordering

$$
\bar{n}<\ldots<\overline{2}<\overline{1}<0_{1}<0_{2}<\ldots<0_{r(r+1) / 2}<1<2 \ldots<n .
$$

Let $w \in S_{r(r+1) / 2}$ be a permutation under this identification, whose RSK insertion tableau is of shape $(r, r-1, \ldots, 1)$. Now for $\alpha \in B_{n}$ let $r$-core representation of $\alpha$ to be

$$
\alpha^{r}=\bar{\alpha}_{n} \ldots \bar{\alpha}_{2} \bar{\alpha}_{1} w \alpha_{1} \alpha_{2} \ldots \alpha_{n}
$$


The algorithm introduced by Barbash and Vogan for $r=0$ and $r=1$ first applies RSK algorithm on $\alpha^{0}$ and respectively $\alpha^{1}$. Then starting from the lowest number $\bar{n}$, it vacates the negative integer $\bar{i}$ in the tableaux by jeu de taquin slides until it becomes adjacent to $i$, where the evacuation is repeated for $\overline{i-1}$ until $i=1$. The following example illustrates this algorithm for $r=1$.

Example 6 For $\alpha=3 \overline{1} 2 \in B_{n}$, we have $\alpha^{1}=\overline{2} 1 \overline{3} 03 \overline{1} 2$ be its 1 -core representation. Then Barbash-Vogan algorithm yields:

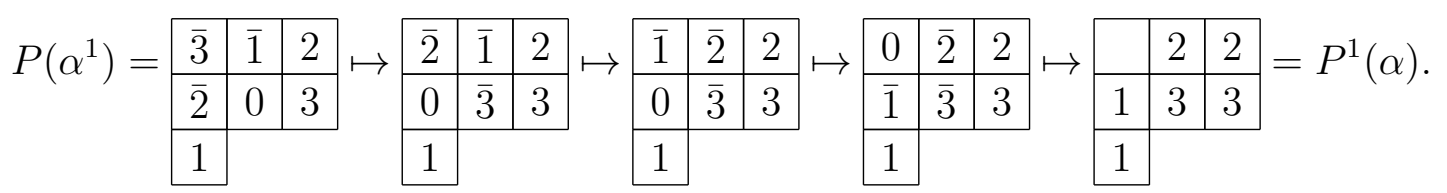

Similarly $Q\left(\alpha^{1}\right)=$\begin{tabular}{|l|l|l|}
\hline$\overline{3}$ & $\overline{2}$ & 1 \\
\hline$\overline{1}$ & 0 & 3 \\
\hline 2 &
\end{tabular}$\mapsto$\begin{tabular}{|l|l|l|}
\hline & 1 & 1 \\
\hline 2 & 3 & 3 \\
\hline 2 &
\end{tabular}$=Q^{1}(\alpha)$

On the other hand by the result of [3], one only needs to apply the same algorithm on $\alpha^{r}$ in order to find $r$-domino tableaux $P^{r}(\alpha)$ and $Q^{r}(\alpha)$ for larger cores.

Theorem 2.6 ([3], Theorem 3.3) Signed permutations $\alpha$ and $\beta$ have the same insertion $r$-domino tableau if and only if $\alpha^{r}$ and $\beta^{r}$ have the same RSK insertion tableau.

The following two propositions can be deduced by using Definition 5, Theorem 2.1, Theorem 2.2 and Theorem 2.6.

Proposition 2.7 Let $\alpha$ be a signed permutation. Then

$$
P^{r}\left(\alpha^{-1}\right)=Q^{r}(\alpha) \text { and } Q^{r}\left(\alpha^{-1}\right)=P^{r}(\alpha) .
$$

Proposition 2.8 Let $\alpha$ and $\beta$ be two signed permutations such that $\alpha^{r} \stackrel{K}{\sim} \beta^{r}$. Then $P^{r}(\alpha)=P^{r}(\beta)$, in other words $\alpha$ and $\beta$ have the same insertion $r$-domino tableau.

\subsection{Descents of domino tableaux and Vogan's map}

Recall that $B_{n}$ carries a Coxeter group structure with the generators $S=\left\{s_{0}, s_{1}, \ldots, s_{n-1}\right\}$ where $\left\{s_{i}=(i, i+1) \mid 1 \leq i \leq n-1\right\}$ is the set of transpositions which also generates the symmetric group $S_{n}$ and $s_{0}$ corresponds to the transposition $(-1,1)$. Let $l(\alpha)$ denote the length of $\alpha$, which is the minimum number of generators of $\alpha$ and let

$$
\begin{aligned}
& \operatorname{Des}_{L}(\alpha):=\left\{i \mid l\left(s_{i} \alpha\right)<l(\alpha) \text { and } 0 \leq i \leq n-1\right\} \\
& =\left\{i \mid \text { if } 1 \leq i \leq n-1 \text { and } i+1 \text { comes before } i \text { in } \alpha^{0}\right\} \\
& \cup\left\{0 \mid \text { if } 1 \text { comes before }-1 \text { in } \alpha^{0}\right\} \\
& \operatorname{Des}_{R}(\alpha):=\operatorname{Des}_{L}\left(\alpha^{-1}\right)
\end{aligned}
$$


denote respectively the sets of left and right descents of $\alpha$.

Now we define the descent set of a $r$-domino tableau $T$ in the following way:

$$
\begin{aligned}
\operatorname{Des}(T):= & \{i \mid \text { if } \operatorname{Dom}(T, i+1) \text { lies below } \operatorname{Dom}(T, i)\} \\
& \cup\{0 \mid \text { if } \operatorname{Dom}(T, 1) \text { is vertical }\}
\end{aligned}
$$

It is a well known property of the RSK algorithm that for a permutation $w \in S_{n}$, we have

$$
\operatorname{Des}_{L}(w)=\operatorname{Des}(P(w))
$$

where $\operatorname{Des}(T):=\{i \mid i+1$ lies below $i$ in $T\}$ is the descent set of a (skew or Young) tableau $T$. Now we have:

Proposition 2.9 For $\alpha \in B_{n}$ we have $\operatorname{Des}_{L}(\alpha)=\operatorname{Des}\left(P^{r}(\alpha)\right)$.

Proof. Observe that $i \in \operatorname{Des}_{L}(\alpha)$ if and only if one of the following

$$
(i+1) i,(\overline{i+1}) i, i(\overline{i+1}), \bar{i}(\overline{i+1})
$$

is a subsequence in $\alpha$. For the first two cases let $S$ denotes the tableau obtained by inserting all the numbers which comes before $i$ in $\alpha$, by Garfinkle's insertion algorithm. Therefore $S$ has a domino cell double labeled by $i+1$. Now since $i$ is inserted horizontally to the first row of $S$ the domino cell labeled by $i+1$ lies below the one labeled by $i$ in $S^{\downarrow i}$, and moreover it remains to be below until the last letter in $\alpha$ inserted, since $i$ and $i+1$ are consecutive numbers. For the last two cases let $T$ be the tableau obtained by inserting all numbers which comes before $i+1$ in $\alpha$. This time domino cell double labeled by $i$ lies in $T$ and since $i+1$ is inserted vertically to the first column of the tableau of $T$, this vertical cell lies below the one labeled by $i$ in $T$. On the other hand insertion of subsequent numbers in $\alpha$ does not change this rule and hence $i \in \operatorname{Des}\left(P^{r}(\alpha)\right)$.

For the reverse inclusion observe that $i \notin \operatorname{Des}_{L}(\alpha)$ if and only if one of the following

$$
i(i+1), \bar{i}(i+1),(i+1) \bar{i},(\overline{i+1}) \bar{i}
$$

is a subsequence in $\alpha$. In this case a similar argument to the one used above shows that $i \notin \operatorname{Des}\left(P^{r}(\alpha)\right)$.

Definition 12 Let $T$ be a $r$-domino tableau and $A$ be a domino corner of shape $(T)$ such that $A=\{(i, j),(i, j+1)\}$ or $A=\{(i, j),(i+1, j)\}$. We denote by $(T, A$, ne $)$ and $(T, A$, ne $)$ the regions of $T$ such that

$$
\begin{aligned}
& (T, A, \text { ne }):=\{(k, l) \mid k<i \text { and } l \geq j\} \\
& (T, A, \mathrm{sw}):=\{(k, l) \mid k \geq i \text { and } l<j\}
\end{aligned}
$$

as illustrated in Figure 1.

Now we are ready to give the following lemma which is crucial in the proof of Theorem 3.2 . 

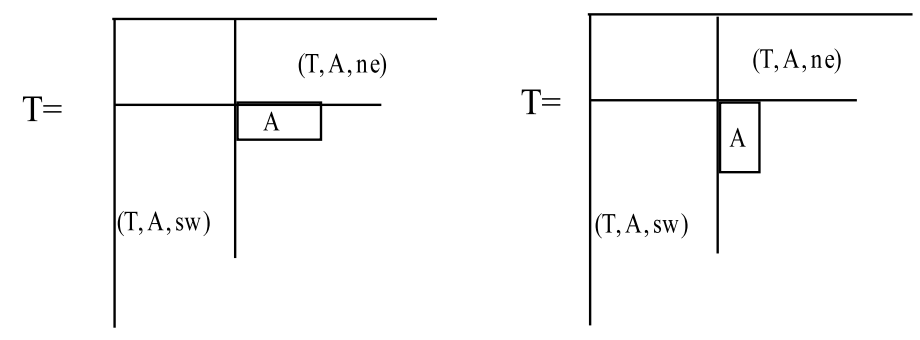

Figure 1:

Lemma 2.10 Let $T$ be a $r$-domino tableau and $A$ be a domino corner of shape $(T)$.

i) Suppose $B$ is a domino corner of shape $\left(T^{\uparrow A}\right)$ which lies in the portion $(T, A, \mathrm{sw})$. Then

$$
\eta\left(T^{\uparrow A \uparrow B}\right)<\eta\left(T^{\uparrow A}\right) .
$$

ii) Suppose $B$ is a domino corner of shape $\left(T^{\uparrow A}\right)$ which lies in the portion (T, $A$, ne). Then

$$
\eta\left(T^{\uparrow A \uparrow B}\right)>\eta\left(T^{\uparrow A}\right) .
$$

Proof. We will just prove the first part of the theorem since the same method applies to the second part. Let $a=\eta\left(T^{\uparrow A}\right), b=\eta\left(T^{\uparrow A \uparrow B}\right)$ and $u$ be a word such that $P^{r}(u)=T^{\uparrow A \uparrow B}$. Then clearly the sign permutation $\alpha=u b a$ has

$$
P^{r}(\alpha)=P^{r}(u b a)=P^{r}(u)^{\downarrow \downarrow \downarrow a}=\left(T^{\uparrow A \uparrow B}\right)^{\downarrow b \downarrow a}=\left(T^{\uparrow A}\right)^{\downarrow a}=T
$$

and

$$
\operatorname{label}\left(Q^{r}(\alpha), A\right)=(n, n) \text { and } \operatorname{label}\left(Q^{r}(\alpha), B\right)=(n-1, n-1) .
$$

On the other hand since $B \in\left(Q^{r}(\alpha), A\right.$, sw $)$ this shows that $n-1 \notin \operatorname{Des}\left(Q^{r}(\alpha)\right)$. Now by Proposition 2.7 and Proposition 2.9 we have

$$
n-1 \notin \operatorname{Des}_{L}\left(\alpha^{-1}\right)=\operatorname{Des}_{R}(\alpha)
$$

and therefore $\alpha_{n-1}=b$ can not be bigger than $\alpha_{n}=a$. Therefore $a=\eta\left(T^{\uparrow A}\right)>b=$ $\eta\left(T^{\uparrow A \uparrow B}\right)$ as desired.

\subsubsection{Vogan's map}

Let $\alpha, \beta \in B_{n}$ whose usual representations satisfy

$$
\begin{aligned}
& \alpha=\alpha_{1} \ldots \alpha_{i-1}\left(\alpha_{i} \alpha_{i+1}\right) \alpha_{i+2} \ldots \alpha_{n} \\
& \beta=\alpha_{1} \ldots \alpha_{i-1}\left(\alpha_{i+1} \alpha_{i}\right) \alpha_{i+2} \ldots \alpha_{n}
\end{aligned}
$$

where either $\alpha_{i}<\alpha_{i+2}<\alpha_{i+1}$ or $\alpha_{i}<\alpha_{i-1}<\alpha_{i+1}$. By extending Definition 5, we say $\alpha$ and $\beta$ are equivalent through single Knuth relation. We write $\alpha \stackrel{K}{\sim} \beta$ in $B_{n}$ if one of 
them can be obtained from the other by applying a sequence of Knuth relations. Clearly $\alpha \stackrel{K}{\sim} \beta$ yields $\alpha^{r} \stackrel{K}{\sim} \beta^{r}$ i.e., Knuth relations which are obtained on the usual representation of signed permutations does not change insertion $r$-domino tableaux. On the other hand their effect on recording tableaux for the case $r=0,1$ is studied by Garfinkle [5, 2.1.102.1.19]. Our following analysis is based on her work, including all notations, definitions and maps. Let $r \geq 0$.

For $i, j$ two adjacent integers satisfying $1 \leq i, j \leq n-1$, consider the following sets:

$$
\begin{aligned}
D_{i, j}\left(B_{n}\right) & :=\left\{\alpha \in B_{n} \mid i \in \operatorname{Des}_{L}(\alpha) \text { but } j \notin \operatorname{Des}_{L}(\alpha)\right\} \\
D_{i, j}\left(S D T^{r}(n)\right) & :=\left\{T \in S D T^{r}(n) \mid i \in \operatorname{Des}(T) \text { but } j \notin \operatorname{Des}(T)\right\}
\end{aligned}
$$

together with the map $V_{i, j}: D_{i, j}\left(B_{n}\right) \mapsto D_{j, i}\left(B_{n}\right)$ where $V_{i, j}(\alpha)=\left\{s_{i} \cdot \alpha, s_{j} \cdot \alpha\right\} \cap D_{j, i}\left(B_{n}\right)$. Also define a map

$$
V_{i, j}: D_{i, j}\left(S D T^{r}(n)\right) \mapsto D_{j, i}\left(S D T^{r}(n)\right)
$$

in the following manner: Without loss of generality we assume that $j>i$, i.e., $j=i+1$. Observe that if $i \in \operatorname{Des}(T)$ but $i+1 \notin \operatorname{Des}(T)$ then $i+1$ lies below $i$ in $T$ whereas $i+2$ lies right to $i+1$ in $T$. On the other hand we have two cases according to the positions of dominos labeled with $(i, i)$ and $(i+2, i+2)$ with respect to each other.

Case 1. We first assume that $i+2$ lies below $i$ in $T$. Since the $i+2$ lies to the right of $i+1$ and $i+1$ lies below $i$ we have two cases to consider: If the boundaries $\operatorname{Dom}(T, i+1)$ and $\operatorname{Dom}(T, i)$ intersect at most at a point then $V_{i, i+1}(T)$ is obtained by interchanging the labels $i$ and $i+1$ in $T$. Otherwise there is only one possibility which satisfies $i+2$ lies below $i$ and it lies to the right of $i+1$, in which $T$ has the subtableau $U$ as illustrated below and $V_{i, i+1}(T)$ is obtained by substituting $U$ with $U^{\prime}$ in $T$.

$$
U=\begin{array}{|c|c|}
\hline i & i \\
\hline i+1 & i+2 \\
\hline i+1 & i+2 \\
\hline
\end{array} \quad U^{\prime}=\begin{array}{|c|c|}
\hline i & i+1 \\
\hline i & i+1 \\
\hline i+2 & i+2 \\
\hline
\end{array}
$$

Case 2. Now we assume $i+2$ lies strictly right to $i$ in $T$. Again if the boundaries of $\operatorname{Dom}(T, i+1)$ and $\operatorname{Dom}(T, i+2)$ intersect at most at a point then $V_{i, i+1}(T)$ is obtained by interchanging the labels $i+1$ and $i+2$ in $T$. Otherwise there is only one possible case where $T$ has the subtableau $U$ given below and $V_{i, i+1}(T)$ is obtained by substituting $U$ with $U^{\prime}$ in $T$.

$$
U=\begin{array}{|c|c|c|}
\hline i & i & i+2 \\
\hline i+1 & i+1 & i+2 \\
\hline
\end{array} \quad U^{\prime}=\begin{array}{|c|c|c|}
\hline i & i+1 & i+1 \\
\hline i & i+2 & i+2 \\
\hline
\end{array}
$$

Example 7 We have $T_{2}=V_{5,6}\left(T_{1}\right), T_{3}=V_{3,4}\left(T_{2}\right)$, and $T_{4}=V_{4,5}\left(T_{3}\right)=V_{6,5}\left(T_{3}\right)$ for the following tableaux.

$$
T_{1}=\begin{array}{|l|l|l|}
\hline 1 & 2 & \mathbf{5} \\
\hline 1 & 2 & \mathbf{5} \\
\hline 3 & 3 & \mathbf{7} \\
\hline 4 & \mathbf{6} & \mathbf{7} \\
\hline 4 & \mathbf{6}
\end{array} \quad T_{2}=\begin{array}{|l|l|l|}
\hline 1 & 2 & 6 \\
\hline 1 & 2 & 6 \\
\hline \mathbf{3} & \mathbf{3} & 7 \\
\hline \mathbf{4} & \mathbf{5} & 7 \\
\hline \mathbf{4} & \mathbf{5}
\end{array} \quad T_{3}=\begin{array}{l|l|l|l|}
\hline 1 & 2 & \mathbf{6} \\
\hline 1 & 2 & \mathbf{6} \\
\hline 3 & \mathbf{4} & \mathbf{7} \\
\hline 3 & \mathbf{4} & \mathbf{7} \\
\hline \mathbf{5} & \mathbf{5}
\end{array} \quad T_{4}=\begin{array}{|l|l|l|}
\hline 1 & 2 & 5 \\
\hline 1 & 2 & 5 \\
\hline 3 & 4 & 7 \\
\hline 3 & 4 & 7 \\
\hline 6 & 6 & \multicolumn{1}{|l}{}
\end{array}
$$


Remark. The map $V_{i, j}$ is first introduced on the symmetric group by Vogan [23], with the aim of classifying the primitive ideals in the universal enveloping algebra of complex semi simple Lie algebras.

Lemma 2.11 Let $i$ and $j$ be two consecutive integers such that $1 \leq i, j \leq n-1$. Suppose $\alpha \in D_{i, j}\left(B_{n}\right)$. Then $P^{r}(\alpha) \in D_{i, j}\left(S D T^{r}(n)\right)$ and

$$
P^{r}\left(V_{i, j}(\alpha)\right)=V_{i, j}\left(P^{r}(\alpha)\right) .
$$

Proof. This result is first proven by Garfinkle [5, Theorem 2.1.19] for $r=0,1$. On the other hand one can check that her proof does not depend on the specific value of $r$ and it can easily be extended for any value of $r$. We omit the proof for the sake of space.

The following result has an important role in the proof of Theorem 3.2.

Corollary 2.12 Let $\alpha=\alpha_{1} \ldots \alpha_{i-1} \alpha_{i} \alpha_{i+1} \alpha_{i+2} \ldots \alpha_{n}$ and $\beta=\alpha_{1} \ldots \alpha_{i-1} \alpha_{i+1} \alpha_{i} \alpha_{i+2} \ldots \alpha_{n}$ be two sign permutation which differ by a single Knuth relation. Then one of the following is satisfied:

1) $\alpha_{i}<\alpha_{i+2}<\alpha_{i+1}$ then $\beta^{-1}=V_{i+1, i}\left(\alpha^{-1}\right)$ and $Q^{r}(\beta)=V_{i+1, i}\left(Q^{r}(\alpha)\right)$.

2) $\alpha_{i}>\alpha_{i+2}>\alpha_{i+1}$ then $\beta^{-1}=V_{i, i+1}\left(\alpha^{-1}\right)$ and $Q^{r}(\beta)=V_{i, i+1}\left(Q^{r}(\alpha)\right)$.

3) $\alpha_{i}<\alpha_{i-1}<\alpha_{i+1}$ then $\beta^{-1}=V_{i-1, i}\left(\alpha^{-1}\right)$ and $Q^{r}(\beta)=V_{i-1, i}\left(Q^{r}(\alpha)\right)$.

4) $\alpha_{i}>\alpha_{i-1}>\alpha_{i+1}$ then $\beta^{-1}=V_{i, i-1}\left(\alpha^{-1}\right)$ and $Q^{r}(\beta)=V_{i, i-1}\left(Q^{r}(\alpha)\right)$.

Proof. Assumptions on $\alpha$ and $\beta$ in the first case yields that $\beta^{-1}=s_{i} \cdot \alpha^{-1} \in D_{i, i+1}\left(B_{n}\right)$ and $\alpha^{-1} \in D_{i+1, i}\left(B_{n}\right)$. Therefore $\beta^{-1}=V_{i+1, i}\left(\alpha^{-1}\right)$ and by Lemma 2.11

$$
Q^{r}(\beta)=P^{r}\left(\beta^{-1}\right)=P^{r}\left(V_{i+1, i}\left(\alpha^{-1}\right)\right)=V_{i+1, i}\left(P^{r}\left(\alpha^{-1}\right)\right)=V_{i+1, i}\left(Q^{r}(\alpha)\right) .
$$

For the other cases the result follows similarly.

\section{Plactic relations for $r$-domino tableaux}

Recall that for any $a \in \mathbb{Z}, \bar{a}$ represents $-a$ if $a>0$ and it represents $|a|$ otherwise.

Definition 13 For $\alpha$ and $\beta$ are two signed permutations in $B_{m}$ and $r \geq 0$, we say $\alpha$ and $\beta$ are $r$-plactic equivalent, $\alpha \stackrel{p_{r}}{\sim} \beta$, if one of them can be obtained from the other by applying a sequence of $\mathrm{D}_{i}^{r}$ relations for $i=1, \ldots 5$, explained below. Moreover, we say $\alpha$ and $\beta$ are $r$-coplactic equivalent, $\alpha \stackrel{p_{r}^{*}}{\sim} \beta$, if $\alpha^{-1} \stackrel{p_{r}}{\sim} \beta^{-1}$.

Let $\alpha=\alpha_{1} \ldots \alpha_{m} \in B_{m}$.

$\mathrm{D}_{1}^{r}$ : If $\alpha_{i}<\alpha_{i+2}<\alpha_{i+1}$ or $\alpha_{i}<\alpha_{i-1}<\alpha_{i+1}$ for some $i \leq m-1$, then

$$
\alpha=\alpha_{1} \ldots \alpha_{i-1}\left(\alpha_{i} \alpha_{i+1}\right) \alpha_{i+2} \ldots \alpha_{m} \sim \alpha_{1} \ldots \alpha_{i-1}\left(\alpha_{i+1} \alpha_{i}\right) \alpha_{i+2} \ldots \alpha_{m}
$$


$\mathrm{D}_{2}^{r}:$ If $r \geq 1$ and if there exists $0<j \leq r$ such that $\alpha_{j}$ and $\alpha_{j+1}$ have opposite signs then

$$
\alpha=\alpha_{1} \ldots \alpha_{j-1}\left(\alpha_{j} \alpha_{j+1}\right) \ldots \alpha_{r+2} \ldots \alpha_{m} \sim \alpha_{1} \ldots\left(\alpha_{j+1} \alpha_{j}\right) \ldots \alpha_{r+2} \ldots \alpha_{m}
$$

$\mathrm{D}_{3}^{r}$ : Suppose that $\left|\alpha_{1}\right|>\left|\alpha_{i}\right|$ for all $2 \leq i \leq r+2$ and $\alpha_{2} \ldots \alpha_{r+2}$ is obtained by concatenating some positive decreasing sequence to the end of some negative increasing sequence (or vice versa), where at least one of the sequences is nonempty. Then

$$
\alpha=\alpha_{1} \alpha_{2} \ldots \alpha_{r+2} \ldots \alpha_{m} \sim \overline{\alpha_{1}} \alpha_{2} \ldots \alpha_{r+2} \ldots \alpha_{m}
$$

$\mathrm{D}_{4}^{r}$ : Let for some $k \geq 1, t=(k+1)(r+k+1) \leq m$ and $\alpha_{1} \ldots \alpha_{t-1}$ can be obtained by concatenating the sequence $\mathfrak{C}_{i+1}$ to the right of $\mathfrak{C}_{i}$, for each $1 \leq i \leq k$, where

$$
\begin{aligned}
& \mathfrak{C}_{i}=a_{i, i+r} \ldots a_{i, 1} b_{i, i} \ldots b_{i, 1} \text { of size } 2 i+r \text { for } 1 \leq i \leq k \\
& \mathfrak{C}_{k+1}=a_{k+1, k+r} \ldots a_{k+1,1} \text { of size } k+r
\end{aligned}
$$

and the integers $a_{i, j}$ and $b_{i, j}$, if exist in $\alpha_{1} \ldots \alpha_{t-1}=\mathfrak{C}_{1} \ldots \mathfrak{C}_{k+1}$, satisfy the following conditions:

$$
\begin{aligned}
& a_{i, j}>0 \text { and } b_{i, j}<0 \text { (or vice versa) } \\
& \left|a_{i, j-1}\right|<\left|a_{i, j}\right|<\left|a_{i+1, j}\right| \text { and }\left|b_{i, j-1}\right|<\left|b_{i, j}\right|<\left|b_{i+1, j}\right| \\
& \left|b_{i, i}\right|<\left|a_{i+1, r+i+1}\right|<\left|b_{i+1, i+1}\right| \text { for all } i=1, \ldots, k-1 .
\end{aligned}
$$

Let $n=\max \left\{\left|\alpha_{1}\right|, \ldots,\left|\alpha_{t-1}\right|\right\}$ and suppose that $\alpha_{t}=z$ satisfies one of the followings:

i. $\left|b_{k, k}\right|=n$ and $z$ is an integer between $a_{k+1,1}$ and $b_{k, 1}$

ii. $\left|a_{k+1, r+k}\right|=n$ and $z$ is an integer between $a_{k, 1}$ and $b_{k, 1}$

iii. $\left|a_{k+1, r+k}\right|=n, z$ is an integer between $a_{k, 1}$ and $a_{k+1,1}$ and $\left|a_{k+1, i}\right|<\left|a_{k, i+1}\right|$ for some $1<i \leq k-1$.

Then we set

$$
\alpha=\ldots \mathfrak{C}_{k} \mathfrak{C}_{k+1} z \alpha_{t+1} \ldots \alpha_{m} \sim \ldots \overline{\mathfrak{C}_{k}} \mathfrak{C}_{k+1} z \alpha_{t+1} \ldots \alpha_{m}
$$

where $\overline{\mathfrak{C}_{k}}=\overline{b_{k, k}} a_{k, k+r} \ldots a_{k, 1} b_{k, k-1} \ldots b_{k, 1}$.

$\mathrm{D}_{5}^{r}$ : Let for some $k \geq 1, t=(k+1)(r+k+2) \leq m$ and $\alpha_{1} \ldots \alpha_{t-1}$ can be obtained by concatenating the sequence $\mathfrak{C}_{i+1}$ to the right of $\mathfrak{C}_{i}$, for each $1 \leq i \leq k$, where

$$
\begin{aligned}
& \mathfrak{C}_{i}=a_{i, i+r} \ldots a_{i, 1} b_{i, i} \ldots b_{i, 1} \text { of size } 2 i+r \text { for } 1 \leq i \leq k \\
& \mathfrak{C}_{k+1}=a_{k+1, k+1+r} \ldots a_{k+1,1} b_{k+1, k} \ldots b_{k+1,1} \text { of size } 2 k+r+1
\end{aligned}
$$


and the integers $a_{i, j}$ and $b_{i, j}$, if exist in $\alpha_{1} \ldots \alpha_{t-1}=\mathfrak{C}_{1} \ldots \mathfrak{C}_{k+1}$, satisfy the following conditions:

$$
\begin{aligned}
& a_{i, j}>0 \text { and } b_{i, j}<0 \text { (or vice versa) } \\
& \left|a_{i, j-1}\right|<\left|a_{i, j}\right|<\left|a_{i+1, j}\right| \text { and }\left|b_{i, j-1}\right|<\left|b_{i, j}\right|<\left|b_{i+1, j}\right| \\
& \left|a_{i, r+i}\right|<\left|b_{i, i}\right|<\left|a_{i+1, r+i+1}\right| \text { for all } i=1, \ldots, k
\end{aligned}
$$

Let $n=\max \left\{\left|\alpha_{1}\right|, \ldots,\left|\alpha_{t-1}\right|\right\}$ and suppose that $\alpha_{t}=z$ satisfies one of the followings:

i. $\left|a_{k+1, r+k+1}\right|=n$ and $z$ is an integer between $a_{k+1,1}$ and $b_{k+1,1}$

ii. $\left|b_{k+1, k}\right|=n$ and $z$ is an integer between $a_{k+1,1}$ and $b_{k, 1}$

iii. $\left|b_{k+1, k}\right|=n, z$ is an integer between $b_{k, 1}$ and $b_{k+1,1}$ and $\left|b_{k+1, i}\right|<\left|b_{k, i+1}\right|$ for some $1<i \leq k-1$.

Then we set

$$
\alpha=\ldots \mathfrak{C}_{k} \mathfrak{C}_{k+1} z \alpha_{t+1} \ldots \alpha_{m} \sim \ldots \overline{\mathfrak{C}_{k}} \overline{\mathfrak{C}_{k+1}} z \alpha_{t+1} \ldots \alpha_{m}
$$

where $\overline{\mathfrak{C}_{k}} \overline{\mathfrak{C}_{k+1}}=a_{k, k+r} \ldots a_{k, 1} \overline{a_{k+1, k+1+r}} b_{k, k} \ldots b_{k, 1} a_{k+1, k+r} \ldots a_{k+1,1} b_{k+1, k} \ldots b_{k+1,1}$.

Following example illustrates $D_{4}^{r}$ for $r=0$, where $k=1$.

Example 8 Consider

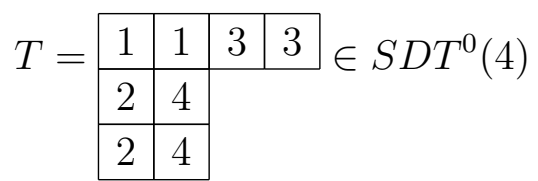

and its domino corners $A=\{(3,1),(3,2)\}, B=\{(2,2),(3,2)\}, C=\{(1,3),(1,4)\}$ together with the sets

$$
\mathcal{K}_{A}(T)=\{143 \overline{2}, 413 \overline{2}, \overline{4} 13 \overline{2}\}, \mathcal{K}_{B}(T)=\{13 \overline{4} \overline{2}, 1 \overline{4} 3 \overline{2}\}, \mathcal{K}_{C}(T)=\{\overline{4} 1 \overline{2} 3,1 \overline{4} \overline{2} 3,41 \overline{2} 3\}
$$

where $\mathcal{K}_{(-)}(T)$ consist of all signed permutations whose insertion gives $T$ with the rule that the last opening domino corner cell in the insertion is (-). One can easily see that permutations in each set above are related by a sequence of $D_{1}^{0}$ and $D_{3}^{0}$ relations. Therefore one needs to obtain some relations between the permutations of these distinct sets. Here $413 \overline{2} \in \mathcal{K}_{A}(T)$ and $41 \overline{2} 3 \in \mathcal{K}_{C}(T)$ are related by $D_{1}^{r}$ relations so a relation which connects either $\mathcal{K}_{A}(T)$ and $\mathcal{K}_{B}(T)$ or $\mathcal{K}_{B}(T)$ and $\mathcal{K}_{C}(T)$ is needed. Now $D_{4}^{0}$ relates $1 \overline{4} 3 \overline{2} \in \mathcal{K}_{B}$ and $413 \overline{2} \in \mathcal{K}_{A}(T)$ under the formulation

$$
1 \overline{4} 3 \overline{2}=\underbrace{a_{11} b_{11}}_{\mathfrak{C}_{1}} \underbrace{a_{21}}_{\mathfrak{C}_{2}} z \text { and } 413 \overline{2}=\underbrace{\overline{b_{11}} a_{11}}_{\overline{\mathfrak{C}_{1}}} \underbrace{a_{21}}_{\mathfrak{C}_{2}} z
$$

where $k=1$ and $r=0$. 
The following remark will be much more clear in the proof (Case 3.2.2) of our main result Theorem 3.2.

Remark. In general consider the tableau $T$ whose shape has the form

$$
(s+i, s+i-1, \ldots, s+1, \underline{s, s-2, s-2, s-4}, s-3, \ldots, 2,1) \text { for some } s \geq 4, i \geq 0
$$

as Figure 2(a) and (b) illustrates. Observe that such a tableau has exactly three domino corners namely

$A=\{(i+3, s-3),(i+3, s-2)\}, B=\{(i+2, s-2),(i+3, s-2)\}, C=\{(i+1, s-1),(i+1, s)\}$

Now if $s+i=r+2 k$ for some $k \geq 1$ then $\mathcal{K}_{A}(T)$ and $\mathcal{K}_{B}(T)$ are related by $D_{4}^{r}$ relations with $a_{i, j}>0$ and $b_{i, j}<0$. On the other hand if $s+i=r+2 k+1$ for some $k \geq 1$ then $\mathcal{K}_{A}(T)$ and $\mathcal{K}_{B}(T)$ are related by $D_{5}^{r}$ relations with $a_{i, j}<0$ and $b_{i, j}>0$.
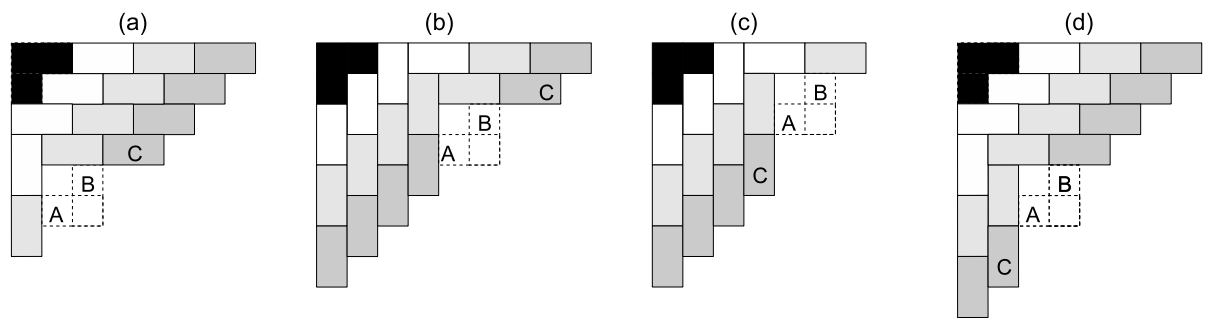

Figure 2:

On the other hand if $T$ has shape

$$
(s+i, s+i-1 \ldots, s+1, s, s, s-2, s-2, s-3, \ldots, 2,1) \text { for some } s \geq 3, i \geq 0,
$$

as Figure 2(c) and (d) illustrates, then $T$ has exactly three domino corners

$A=\{(i+2, s-1),(i+2, s)\}, B=\{(i+1, s),(i+2, s)\}, C=\{(i+3, s-2),(i+3, s-2)\}$

Now if $s+i=r+2 k$ for some $k \geq 1$ then $\mathcal{K}_{A}(T)$ and $\mathcal{K}_{B}(T)$ are related by $D_{5}^{r}$ relations with $a_{i, j}>0$ and $b_{i, j}<0$. On the other hand if $s+i=r+2 k+1$ for some $k \geq 1$ then $\mathcal{K}_{A}(T)$ and $\mathcal{K}_{B}(T)$ are related by $D_{4}^{r}$ relations with $a_{i, j}<0$ and $b_{i, j}>0$.

Here it is natural to ask whether some simpler relations exist for the tableaux presented above. In fact we know a rule which relates $\mathcal{K}_{B}(T)$ and $\mathcal{K}_{C}(T)$, but its formal description requires four relations, yet they appear to us not handy when it comes to proving our main result.

Theorem 3.1 If $\alpha \stackrel{p_{r}}{\sim} \beta$ in $B_{m}$ then they have the same insertion $r$-domino tableaux.

Proof. For the proof of the theorem it will be enough to consider the case when $\alpha$ and $\beta$ differ by a single $\mathrm{D}_{i}^{r}$ relation for $i=1, \ldots, 5$. 
Observe that $\mathrm{D}_{1}^{r}$ is just the single Knuth relation defined on the usual representation of signed permutations and therefore result follows from Proposition 2.8.

For $\mathrm{D}_{2}^{r}, r \geq 1$, let $\alpha_{j}$ and $\alpha_{j+1}$ have opposite signs in $\alpha=\alpha_{1} \ldots \alpha_{j} \alpha_{j+1} \ldots \alpha_{r+2} \ldots \alpha_{m}$ for some $j \leq r$. Observe that the size of empty corners of $r$-staircase shape is $r+1$. Let $S$ denote the tableau obtained by inserting first $j-1$ elements of $\alpha$ in to $r$-staircase shape. If $r=1$ then $\alpha_{1} \ldots \alpha_{j-1}$ is empty and one can check easily that insertion of $\alpha_{j} \alpha_{j+1}$ and $\alpha_{j+1} \alpha_{j}$ into 1-staircase shape creates the same tableau. For $r>1$, observe that since $j+1 \leq r+1$, the insertion of $\alpha_{1} \ldots \alpha_{j} \alpha_{j+1}$ creates two connected union of domino cells, where one consists of horizontal domino cells labeled by the positive numbers in $\alpha_{1} \ldots \alpha_{j+1}$, concatenated to the right of $r$-staircase shape and the other consists of vertical domino cells labeled by the absolute value of negative numbers in the same sequence, concatenated below $r$-staircase shape. Without loss of generality we assume $\alpha_{j}>0$ and $\alpha_{j+1}<0$ and let $A$ and $B$ denote the horizontal and vertical domino cells appearing after the insertion of $\alpha_{j}$ and $\alpha_{j+1}$ respectively as illustrated in Figure 3 for $r=6$ and $j=6$.
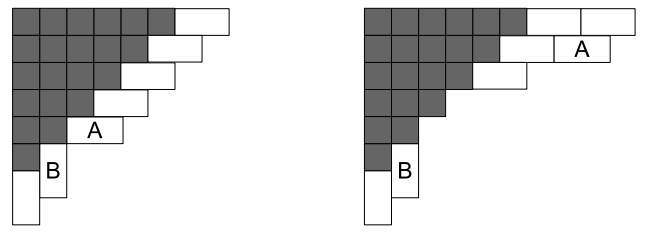

Figure 3:

Now one can easily see that changing the order of $\alpha_{j} \alpha_{j+1}$ in $\alpha$ still gives the same tableau, since $\alpha_{j}$ and $\alpha_{j+1}$ have opposite signs and $j+1 \leq r+1$.

For $\mathrm{D}_{3}^{r}$, let $\left|\alpha_{1}\right|>\left|\alpha_{i}\right|$ for all $2 \leq i \leq r+2$ and suppose that for some $k \geq 0, l \geq 0$ and $k+l=r+1$

$$
\alpha_{2} \ldots \alpha_{r+2}=x_{1} \ldots x_{k} y_{1} \ldots y_{l}
$$

where $x_{1} \ldots x_{k}$ is a positive decreasing and $y_{1} \ldots y_{l}$ is a negative increasing sequence (or vice versa). Here observe that we can not have both $k=0$ and $l=0$, since then $x_{1} \ldots x_{k} y_{1} \ldots y_{l}$ is empty but $\alpha_{2} \ldots \alpha_{r+2}$ is not, even if $r=0$. So without loss of generality we assume that $l \geq 1$. If $r=0$, we must have $k=0, l=1$ and $\alpha_{2}=y_{1}$ and one can easily check that in this case $\alpha_{1} \alpha_{2}$ and $\overline{\alpha_{1}} \alpha_{2}$ give the same tableau. For $r>0$ the insertion of $\alpha_{1} x_{1} \ldots x_{k} y_{1} \ldots y_{l-1}$ and $\overline{\alpha_{1}} x_{1} \ldots x_{k} y_{1} \ldots y_{l-1}$ yields two tableaux which differ by only the position of the domino cell $\{(k+1, l),(k+1, l+1)\}$ and $\{(k+1, l),(k+2, l)\}$, labeled by $\left(\left|\alpha_{1}\right|,\left|\alpha_{1}\right|\right)$, as illustrated in Figure 4 for $r=5$. On the other hand the insertion of $y_{l}$ in both tableaux yields the same tableau.
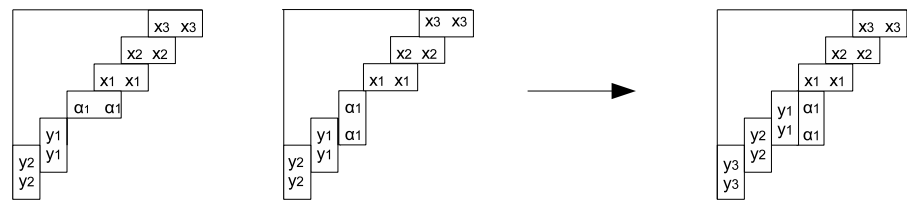

Figure 4:

In the following we will just deal with the relation $\mathrm{D}_{4}^{r}$ since then the same method also applies to the relation $\mathrm{D}_{5}^{r}$. 
It is enough to consider the case where $\alpha=\ldots \mathfrak{C}_{k} \mathfrak{C}_{k+1} z$ and $\beta=\ldots \overline{\mathfrak{C}_{k}} \mathfrak{C}_{k+1} z$ as described in $\mathrm{D}_{4}^{r}$. Observe that in this case we have $m=n$. On the other hand, since the tableau obtained by taking all $a_{i, j}$ 's negative and $b_{i, j}$ positive is the transpose of the tableau obtained by otherwise, it is enough to consider only one case. So let

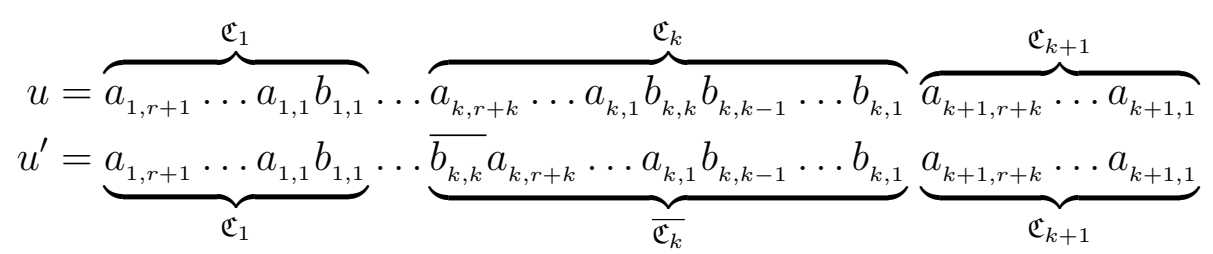

where $a_{i, j}>0$ and $b_{i, j}<0$ above and let $S=P(u)$ and $T=P\left(u^{\prime}\right)$.

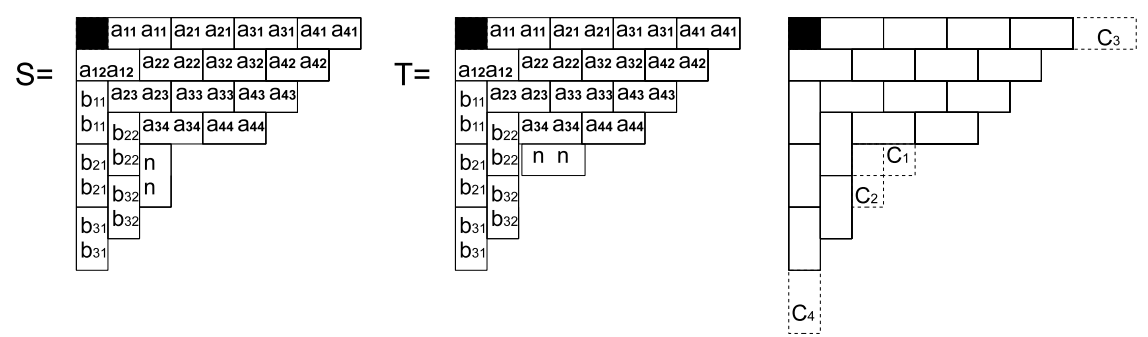

Figure 5:

We first assume that $b_{k, k}=\bar{n}$ and $b_{k, 1}<z<a_{k+1,1}$. Then $S$ and $T$ differ only by the domino cell double labeled by $n$ as illustrated in Figure 5 for $r=1$ and $k=3$ (where the absolute value on $b_{i, j}$ is removed for the sake of simplicity). In other words $S_{<n}=T_{<n}$ with shape

$$
(s, s-1, \ldots, s-k, s-k-4, s-k-4, s-k-4, s-k-5, \ldots, 2,1)
$$

for some $s=r+2 k+2$. Therefore $S_{<n}=T_{<n}$ has exactly four empty domino corners:

$$
\begin{aligned}
& C_{1}=\{(k+1, k),(k+1, k+1)\} \\
& C_{2}=\{(k+1, k),(k+2, k)\} \\
& C_{3}=\{(1, s+1),(1, s+2)\} \\
& C_{4}=\{(s, 1),(s+1,1)\} .
\end{aligned}
$$

Recall that by Lemma 2.4 one has

$$
S^{\downarrow z}=\left(S_{<n}\right)^{\downarrow z} \leftarrow \operatorname{Dom}(S, n) \text { and } T^{\downarrow z}=\left(T_{<n}\right)^{\downarrow z} \leftarrow \operatorname{Dom}(T, n)
$$

where $\operatorname{Dom}(S, n)=\left[C_{2},(n, n)\right]$ and $\operatorname{Dom}(T, n)=\left[C_{1},(n, n)\right]$. On the other hand the assumption

$$
b_{k, 1}<z<a_{k+1,1}
$$

yields two choices for the new domino cell appearing in $\left(S_{<n}\right)^{\downarrow z}=\left(T_{<n}\right)^{\downarrow z}$, which are $C_{1}$ and $C_{2}$. Now one can observe that whether $C_{1}$ or $C_{2}$ appears sliding $\operatorname{Dom}(S, n)$ and 
$\operatorname{Dom}(T, n)$ over $\left(S_{<n}\right)^{\downarrow z}=\left(T_{<n}\right)^{\downarrow z}$ gives the same tableau. Therefore $P^{r}(u z)=P^{r}\left(u^{\prime} z\right)$ as desired.

Now assume $a_{k+1, r+k}=n$. This time $S=P(u)$ and $T=P\left(u^{\prime}\right)$ differ only by the domino cell double labeled by $\left|b_{k, k}\right|$, as illustrated in Figure 6 (a) and (b) for $r=1$ and $k=3$.
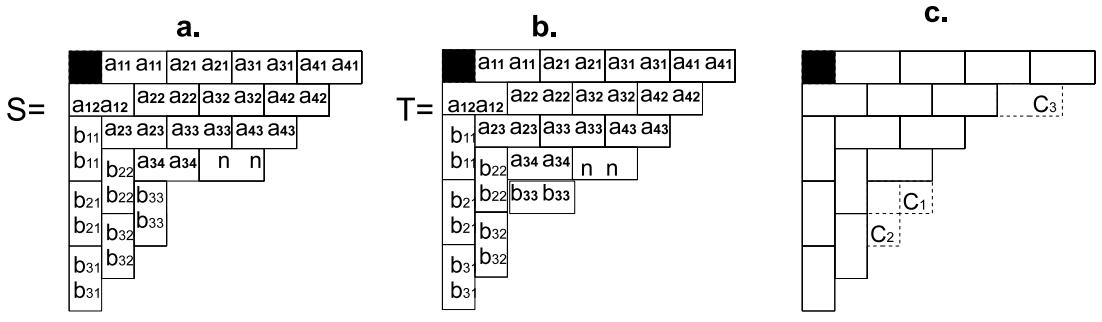

Figure 6:

Let $\mathcal{A}$ be the set of labels which are greater than or equal to $\left|b_{k, k}\right|$. Therefore

$$
\mathcal{A}=\left\{\left|b_{k, k}\right|, a_{k+1, i}, \ldots, a_{k+1, r+k-1}, a_{k+1, r+k}=n\right\}
$$

for some $1 \leq i \leq r+k$. Consider the tableaux $S^{\prime}=S_{<\left|b_{k, k}\right|}$ and $T^{\prime}=T_{<\left|b_{k, k}\right|}$ which are obtained by erasing from $S$ and $T$ respectively the domino cells double labeled by $\mathcal{A}$. Now $S^{\prime}=T^{\prime}$ and moreover $\left(S^{\prime}\right)^{\downarrow z}=\left(T^{\prime}\right)^{\downarrow z}$. Recall that under the assumption $a_{k+1, r+k}=n$ we have

$$
\begin{aligned}
& \text { either } b_{k, 1}<z<a_{k, 1} \\
& \text { or } a_{k, 1}<z<a_{k+1,1} \text { and } a_{k+1, i}<a_{k, i+1} \text { for some } 1<i \leq k-1 .
\end{aligned}
$$

Observe that in case $b_{k, 1}<z<a_{k, 1}$ we have two choices for the new domino cell appearing in $\left(S^{\prime}\right)^{\downarrow z}=\left(T^{\prime}\right)^{\downarrow z}$ which are, as illustrated in Figure 6 (c),

$$
C_{1}=\{(k+1, k),(k+1, k+1)\}, C_{2}=\{(k+1, k),(k+2, k)\} .
$$

On the other hand if $a_{k, 1}<z<a_{k+1,1}$ one would add the domino cell $C_{3}=\{(i, r+2 k+$ $1),(i, r+2 k+2)\}$ in the above list but existence of $C_{3}$ requires $a_{k, 1}<z<a_{k+1,1}$ and $a_{k+1, i} \nless a_{k, i+1}$ for any $1<i \leq k-1$. Therefore in both cases $C_{1}$ and $C_{2}$ are the only choices for a new domino cell appearing in $\left(S^{\prime}\right)^{\downarrow z}=\left(T^{\prime}\right)^{\downarrow z}$.

Recall that

$$
\begin{aligned}
& S^{\downarrow z}=\left(S^{\prime}\right)^{\downarrow z} \leftarrow \operatorname{Dom}\left(S,\left|b_{k, k}\right|\right) \leftarrow \operatorname{Dom}\left(S, a_{k+1, i}\right) \ldots \leftarrow \operatorname{Dom}\left(S, a_{k+1, r+k-1}\right) \leftarrow \operatorname{Dom}(S, n) \\
& T^{\downarrow z}=\left(T^{\prime}\right)^{\downarrow z} \leftarrow \operatorname{Dom}\left(T,\left|b_{k, k}\right|\right) \leftarrow \operatorname{Dom}\left(T, a_{k+1, i}\right) \ldots \leftarrow \operatorname{Dom}\left(T, a_{k+1, r+k-1}\right) \leftarrow \operatorname{Dom}(T, n)
\end{aligned}
$$

where $\operatorname{Dom}\left(S,\left|b_{k, k}\right|\right)=\left[C_{2},\left(\left|b_{k, k}\right|,\left|b_{k, k}\right|\right)\right]$ and $\operatorname{Dom}\left(T,\left|b_{k, k}\right|\right)=\left[C_{1},\left(\left|b_{k, k}\right|,\left|b_{k, k}\right|\right)\right]$. Now whichever $C_{1}$ or $C_{2}$ appears in $\left(S^{\prime}\right)^{\downarrow z}=\left(T^{\prime}\right)^{\downarrow z}$, sliding the domino cell double labeled by $\left|b_{k, k}\right|$ gives the same tableau, i.e.,

$$
\left(S^{\prime}\right)^{\downarrow z} \leftarrow \operatorname{Dom}\left(S,\left|b_{k, k}\right|\right)=\left(T^{\prime}\right)^{\downarrow z} \leftarrow \operatorname{Dom}\left(T,\left|b_{k, k}\right|\right) .
$$

Moreover since $\operatorname{Dom}\left(S, a_{k+1, j}\right)=\operatorname{Dom}\left(T, a_{k+1, j}\right)$ for all $i \leq j \leq r+k$ we have $P^{r}(u z)=$ $S^{\downarrow z}=T^{\downarrow z}=P^{r}\left(u^{\prime} z\right)$ as desired. 
Theorem 3.2 If $\alpha$ and $\beta$ have the same insertion $r$-domino tableaux then $\alpha \stackrel{p_{r}}{\sim} \beta$ in $B_{n}$.

Proof. We will proceed by induction. If $n=1$ there is nothing to prove, so suppose that $n>1$ and the statement holds for all signed permutations of size $n-1$.

Let $\alpha=\alpha_{1} \ldots \alpha_{n-1} \alpha_{n}$ and $\beta=\beta_{1} \ldots \beta_{n-1} \beta_{n}$ satisfies $T=P^{r}(\alpha)=P^{r}(\beta)$. Therefore there exist two domino corners say $A$ and $B$ of $T$ such that

$$
\begin{aligned}
& T^{\uparrow A}=P^{r}\left(\alpha_{1} \ldots \alpha_{n-1}\right) \text { and } \eta\left(T^{\uparrow A}\right)=\alpha_{n} \\
& T^{\uparrow B}=P^{r}\left(\beta_{1} \ldots \beta_{n-1}\right) \text { and } \eta\left(T^{\uparrow B}\right)=\beta_{n} .
\end{aligned}
$$

In the following we suppose that $\min (A)$ lies below $\min (B)$.

Cases 1: $A=B$. Then clearly $T^{\uparrow A}=T^{\uparrow B}$ and $\alpha_{n}=\eta\left(T^{\uparrow A}\right)=\eta\left(T^{\uparrow B}\right)=\beta_{n}$. Then since

$$
P\left(\alpha_{1} \ldots \alpha_{n-1}\right)=T^{\uparrow A}=T^{\uparrow B}=P\left(\beta_{1} \ldots \beta_{n-1}\right)
$$

$\alpha_{1} \ldots \alpha_{n-1} \stackrel{p_{r}}{\sim} \beta_{1} \ldots \beta_{n-1}$ by induction and therefore $\alpha=\alpha_{1} \ldots \alpha_{n-1} \alpha_{n} \stackrel{p_{r}}{\sim} \beta_{1} \ldots \beta_{n-1} \beta_{n}=\beta$ as desired.

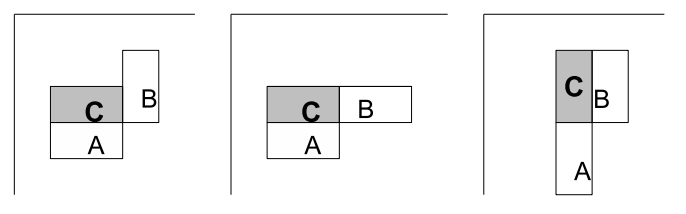

Figure 7: Some illustrations for Case 2

Cases 2: $A \neq B$ and $(T, A, \mathrm{ne}) \cap(T, B, \mathrm{sw})$ contains a domino corner, say $C$ as illustrated in Figure 7. Let

$$
\begin{aligned}
& b=\eta\left(T^{\uparrow A \uparrow B}\right) \\
& c=\eta\left(T^{\uparrow A \uparrow B \uparrow C}\right)
\end{aligned}
$$

and let $\tilde{u}$ be a signed word such that $P^{r}(\tilde{u})=T^{\uparrow A \uparrow B \uparrow C}$. Therefore

$$
P^{r}\left(\tilde{u} c b \alpha_{n}\right)=P^{r}(\tilde{u})^{\downarrow c \downarrow b \downarrow \alpha_{n}}=\left(T^{\uparrow A \uparrow B \uparrow C}\right)^{\downarrow c \downarrow b \downarrow \alpha_{n}}=\left(T^{\uparrow A \uparrow B}\right)^{\downarrow b \downarrow \alpha_{n}}=\left(T^{\uparrow A}\right)^{\downarrow \alpha_{n}}=T
$$

and by induction hypothesis $\tilde{u} c b \stackrel{p_{r}}{\sim} \alpha_{1} \ldots \alpha_{n-1}$ since $P^{r}(\tilde{u} c b)=T^{\uparrow A}=P^{r}\left(\alpha_{1} \ldots \alpha_{n-1}\right)$. Therefore

$$
\tilde{u} c b \alpha_{n} \stackrel{p_{r}}{\sim} \alpha
$$

Observe that since $P^{r}(\tilde{u})=T^{\uparrow A \uparrow B \uparrow C}$, the recording tableau $Q^{r}\left(\tilde{u} c b \alpha_{n}\right)$ has its domino cells $A, B$ and $C$ labeled with $(n, n),(n-1, n-1)$ and $(n-2, n-2)$ respectively.

On the other hand having $B$ in $(T, A$, ne $)$ and $C$ in $(T, B, \mathrm{sw})$ yields by Lemma 2.10 that

$$
b=\eta\left(T^{\uparrow A \uparrow B}\right)>\eta\left(T^{\uparrow A}\right)=\alpha_{n} \text { and } b=\eta\left(T^{\uparrow A \uparrow B}\right)>\eta\left(T^{\uparrow A \uparrow B \uparrow C}\right)=c .
$$

Therefore by Corollary 2.12,

either $b>\alpha_{n}>c$, and hence $u=\tilde{u} c b \alpha_{n} \stackrel{\mathrm{D}_{1}^{r}}{\sim} \tilde{u} b c \alpha_{n}=w$ and $V_{n-1, n-2}\left(Q^{r}(u)\right)=Q^{r}(w)$ or $b>c>\alpha_{n}$, and hence $u=\tilde{u} c b \alpha_{n} \stackrel{\mathrm{D}_{1}^{r}}{\sim} \tilde{u} c \alpha_{n} b=w$ and $V_{n-1, n-2}\left(Q^{r}(u)\right)=Q^{r}(w)$ 
The last argument implies that in both cases the recording tableau $Q^{r}(w)$ of the signed permutation $w$ is obtained by interchanging the labels $(n, n)$ of $A$ and $(n-1, n-1)$ of $B$ in $Q^{r}(u)$ which means that $Q^{r}(w)$ has its domino corner $B$ labeled with $(n, n)$. So we have

$$
P^{r}\left(w_{1} \ldots w_{n-1}\right)=T^{\uparrow B}=P^{r}\left(\beta_{1} \ldots \beta_{n-1}\right) \text { and } w_{n}=\beta_{n} .
$$

Now by induction, $w_{1} \ldots w_{n-1} \stackrel{p_{r}}{\sim} \beta_{1} \ldots \beta_{n-1}$ and therefore $w \stackrel{p_{r}}{\sim} \beta$. Hence $\alpha \stackrel{p_{r}}{\sim} u \stackrel{p_{r}}{\sim} w \stackrel{p_{r}}{\sim} \beta$ as required.

Case 3: $A \neq B,(T, A$, ne $) \cap(T, B, \mathrm{sw})$ is a staircase shape $(s, s-1, \ldots, 1)$ for $s \geq 1$ and $A \cap B$ is a single box. The condition $A \cap B$ is a single box forces that $s=1$. There are several subcases.

Case 3.1: We assume that $T$ has no domino corner beyond $A$ and $B$. Let $\lambda$ and $\lambda^{\prime}$ be respectively the smallest and the largest rectangular box in shape $(T)$ containing both $A$ and $B$, whose east and south boundary coincides with the boundary of shape $(T)$. Then clearly $\lambda=(2,2)$ and we have either $\lambda \subsetneq \lambda^{\prime}$ or $\lambda=\lambda^{\prime}$ as illustrated in Figure 8 .
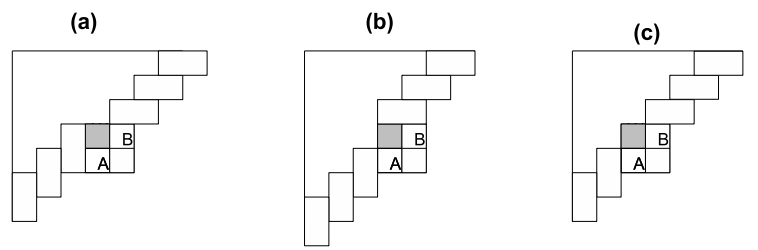

Figure 8: Case 3.1: $T$ has no domino corner beyond $A$ and $B$ and the partitions $(3,3)$, $(2,2,2)$ and $(2,2)$ determine $\lambda^{\prime}$ in (a),(b) and (c) respectively.

Case 3.1.1: We first suppose that $\lambda \subsetneq \lambda^{\prime}$ as illustrated in Figure 8(a) and $(b)$. Since the other case can be dealt with in the same manner after taking the transpose of $T$, below we just consider the case Figure $8(a)$, where there exists a vertical domino cell to the left of $\lambda$ in $\lambda^{\prime}$.
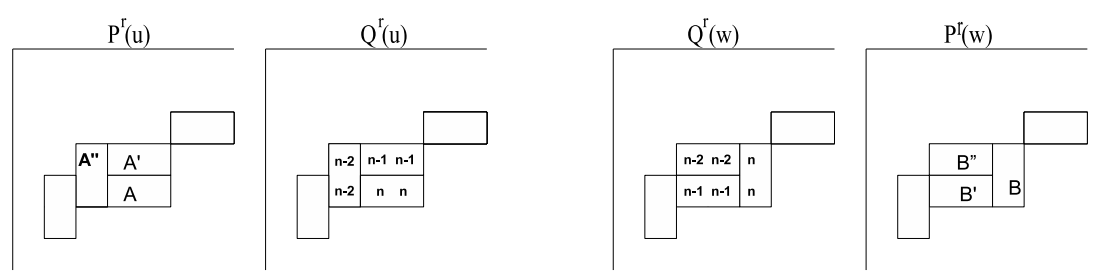

Figure 9: Case 3.1.1

Now observe through Figure 9 that we have a domino corner $A^{\prime}$ of $T^{\uparrow A}$ and $A^{\prime \prime}$ of $T^{\uparrow A \uparrow A^{\prime}}$ as given in Figure 9. Let $a^{\prime}=\eta\left(T^{\uparrow A \uparrow A^{\prime}}\right)$ and $a^{\prime \prime}=\eta\left(T^{\uparrow A \uparrow A^{\prime} \uparrow A^{\prime \prime}}\right)$. Suppose $\tilde{u}$ be a signed word such that $P^{r}(\tilde{u})=T^{\uparrow A \uparrow A^{\prime} \uparrow A^{\prime \prime}}$. Then the signed permutation $u=\tilde{u} a^{\prime \prime} a^{\prime} \alpha_{n}$ has $P^{r}(u)=T$ whereas its recording tableau $Q^{r}(u)$ must have the form as it is shown in Figure 9. 
On the other hand having $A^{\prime}$ in $\left(T, A\right.$, ne) and $A^{\prime \prime}$ in $\left(T, A^{\prime}\right.$, sw $)$ yields by Lemma 2.10 that

$$
a^{\prime}=\eta\left(T^{\uparrow A \uparrow A^{\prime}}\right)>\eta\left(T^{\uparrow A}\right)=\alpha_{n} \quad \text { and } \quad a^{\prime}=\eta\left(T^{\uparrow A \uparrow A^{\prime}}\right)>\eta\left(T^{\uparrow A \uparrow A^{\prime} \uparrow A^{\prime \prime}}\right)=a^{\prime \prime} .
$$

Therefore we have

$$
\begin{aligned}
& \text { either } a^{\prime \prime}<\alpha_{n}<a^{\prime} \text {, so } u=\tilde{u} a^{\prime \prime} a^{\prime} \alpha_{n} \stackrel{\mathrm{D}_{1}^{r}}{\sim} \tilde{u} a^{\prime} a^{\prime \prime} \alpha_{n}=w \text { and } Q^{r}(w)=V_{n-2, n-1}\left(Q^{r}(u)\right) \\
& \text { or } \alpha_{n}<a^{\prime \prime}<a^{\prime}, \quad \text { so } u=\tilde{u} a^{\prime \prime} a^{\prime} \alpha_{n} \stackrel{\mathrm{D}_{1}^{r}}{\sim} \tilde{u} a^{\prime \prime} \alpha_{n} a^{\prime}=w \text { and } Q^{r}(w)=V_{n-2, n-1}\left(Q^{r}(u)\right) .
\end{aligned}
$$

In both cases Corollary 2.12 yields that the recording tableau $Q^{r}(w)$ of $w$ has the form in Figure 9.

Now since $P^{r}\left(\tilde{u} a^{\prime \prime} a^{\prime}\right)=T^{\uparrow A}=P^{r}\left(\alpha_{1} \ldots \alpha_{n-1}\right)$, we have $\tilde{u} a^{\prime \prime} a^{\prime} \stackrel{p_{r}}{\sim} \alpha_{1} \ldots \alpha_{n-1}$ by induction. Therefore

$$
u=\tilde{u} a^{\prime \prime} a^{\prime} \alpha_{n} \stackrel{p_{r}}{\sim} \alpha_{1} \ldots \alpha_{n-1} \alpha_{n}=\alpha .
$$

Similarly, $P^{r}\left(w_{1} \ldots w_{n-1}\right)=T^{\uparrow B}=P^{r}\left(\beta_{1} \ldots \beta_{n-1}\right)$, so $w_{1} \ldots w_{n-1} \stackrel{p_{r}}{\sim} \beta_{1} \ldots \beta_{n-1}$ by induction. On the other hand $w_{n}=\beta_{n}$ and we have $w_{1} \ldots w_{n-1} \beta_{n} \stackrel{p_{r}}{\sim} \beta_{1} \ldots \beta_{n-1} \beta_{n}=\beta$. Hence

$$
\alpha \stackrel{p_{r}}{\sim} u \stackrel{\mathrm{D}_{1}^{r}}{\sim} w \stackrel{p_{r}}{\sim} \beta
$$

Case 3.1.2: No we suppose that $\lambda=\lambda^{\prime}$ as illustrated in Figure 8(c). Observe through Figure 10 that the grey area in the first tableau has a staircase shape and since there are no other domino corners of $T$, we must have either $A$ or $B$ labeled by $(n, n)$.
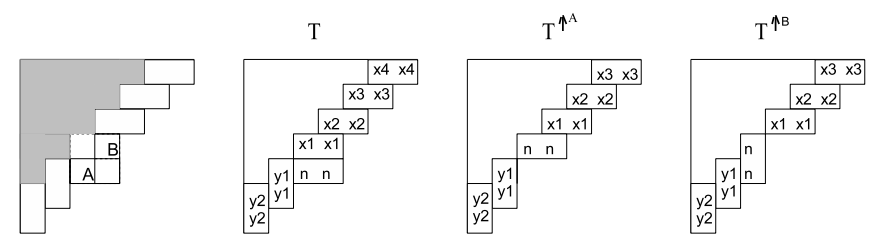

Figure 10: Case 3.1.2

Suppose that the horizontal domino cell $A$ is labeled by $(n, n)$ (The other case can be also dealt with taking the transpose of the tableau). So as Figure 10 illustrates, we have only the horizontal domino cells double labeled by $n, x_{1}, \ldots, x_{k}$ and the vertical domino cells double labeled by $y_{1}, \ldots, y_{l}$ where $k \geq 0, l \geq 0$ and $k+l=r+1$. Therefore at least one of $k$ and $l$ must be nonzero. Without loss of generality assume that $k \geq 1$. In this case observe that $\eta\left(T^{\uparrow A}\right)=\eta\left(T^{\uparrow B}\right)=x_{k}>0$ and this yields $\alpha_{n}=\beta_{n}=x_{k}$. Let $\tilde{u}=\overline{y_{1}} \ldots \overline{y_{l}} x_{1} \ldots x_{k-1}$. Then clearly $P^{r}\left(n \tilde{u} x_{k}\right)=T=P^{r}\left(\bar{n} \tilde{u} x_{k}\right)$ and $n \tilde{u} x_{k} \stackrel{\mathrm{D}_{3}^{r}}{\sim} \tilde{u} \tilde{u} x_{k}$. On the other hand $P^{r}(n \tilde{u})=T^{\uparrow A}$ and $P^{r}(\bar{n} \tilde{u})=T^{\uparrow B}$ and by induction hypothesis we have $n \tilde{u} \stackrel{p_{r}}{\sim} \alpha_{1} \ldots \alpha_{n-1}$ and $\bar{n} \tilde{u} \stackrel{p_{r}}{\sim} \beta_{1} \ldots \beta_{n-1}$. Hence

$$
\alpha=\alpha_{1} \ldots \alpha_{n-1} x_{k} \stackrel{p_{r}}{\sim} n \tilde{u} x_{k} \stackrel{\mathrm{D}_{3}^{r}}{\sim} \bar{n} \tilde{u} x_{k} \stackrel{p_{r}}{\sim} \beta_{1} \ldots \beta_{n-1} x_{k}=\beta .
$$


Case 3.2: Suppose that $T$ has another domino corner, say $C$. Then $C$ must lie either in $(T, B$, ne) or $(T, A, \mathrm{sw})$. Below we assume that $C$ lies in $(T, B$, ne), since the other case can be dealt with in the same manner by considering the transpose of $T$.

Case 3.2.1: We first suppose that $(T, C, \mathrm{sw}) \cap(T, B, \mathrm{ne})$ contains a domino corner as illustrated in Figure 11. Let $\sigma$ be a permutation such that $T^{\uparrow C}=P^{r}\left(\sigma_{1} \ldots \sigma_{n-1}\right)$ and $\eta\left(T^{\uparrow C}\right)=\sigma_{n}$. Therefore $B$ and $C$ satisfy Case 2 and so $\beta \stackrel{p_{r}}{\sim} \sigma$. On the other hand $(\mathrm{T}, \mathrm{C}, \mathrm{sw}) \cap(T, A, \mathrm{ne})$ contains a domino corner, i.e., $A$ and $C$ also satisfy Case 2 and this gives $\alpha \stackrel{p_{r}}{\sim} \sigma$. Hence $\beta \stackrel{p_{r}}{\sim} \alpha$.

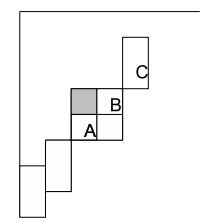

Figure 11: Case 3.2.1

Case 3.2.2: Now we suppose that $C$ satisfies

$$
(T, C, \mathrm{sw}) \cap(T, B, \mathrm{ne})=(s, s-1, \ldots, 2,1) \text { for some } s \geq 1 \text {. }
$$

Further we can assume that $C$ is the only domino corner lying in $(T, B$, ne), since any other domino corner in $(T, B$, ne) must satisfy Case 3.2.1. Below, Figure 12 illustrates the possible subcases:

(a)

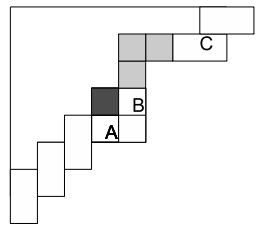

(b)

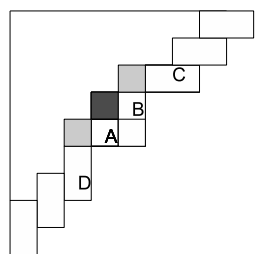

(c)

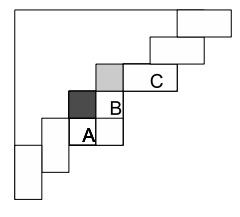

Figure 12: Case 3.2.2

We first suppose that $(T, C, \mathrm{sw}) \cap(T, B$, ne $)$ is a staircase shape $(s, s-1, \ldots, 1)$ for $s>1$ as illustrated in Figure 12(a). Then whether $(T, A$, sw) contains another domino corner or not, $A$ and $B$ are contained in a rectangular shape which is strictly larger than $\lambda=(2,2)$. Therefore this case is similar to the one studied in Case 3.1.1., and the same method applied there gives $\alpha \stackrel{p_{r}}{\sim} \beta$.

Now suppose that $(T, C, \mathrm{sw}) \cap(T, B$, ne) is a single box and that there exist a domino corner, say $D$ in $(T, A, \mathrm{sw})$ as illustrated in Figure $12(b)$. Observe that all $(T, C, \mathrm{sw}) \cap$ $(T, A, \mathrm{ne})$ and $(T, D, \mathrm{ne}) \cap(T, B, \mathrm{sw})$ and $(T, C, \mathrm{sw}) \cap(T, D, \mathrm{ne})$ contain a domino corner i.e., the pair $A$ and $C$, the pair $B$ and $D$ and the pair $C$ and $D$ satisfy Case 2. Let $\sigma$ and $\delta$ be two permutations in $B_{n}$ satisfying:

$$
T^{\uparrow C}=P^{r}\left(\sigma_{1} \ldots \sigma_{n-1}\right), \eta\left(T^{\uparrow C}\right)=\sigma_{n} \text { and } T^{\uparrow D}=P^{r}\left(\delta_{1} \ldots \delta_{n-1}\right), \eta\left(T^{\uparrow D}\right)=\delta_{n} .
$$


Therefore $\alpha \stackrel{p_{r}}{\sim} \sigma \stackrel{p_{r}}{\sim} \delta \stackrel{p_{r}}{\sim} \beta$ as required.

Lastly we suppose that $(T, C, \mathrm{sw}) \cap(T, B$, ne) is a single box and that there exist no domino corners in $(T, A, \mathrm{sw})$ as illustrated in Figure 12(c) (See also Figure 2 for possible other variations, including the transpose of $T$ ). Therefore $T$ has shape

$$
(s+i, s+i-1, \ldots, s+1, s, s-2, s-2, s-4, s-3, \ldots, 2,1) \text { for some } s \geq 4, i \geq 0
$$

and it has three domino corners

$A=\{(i+3, s-3),(i+3, s-2)\}, B=\{(i+2, s-2),(i+3, s-2)\}, C=\{(i+1, s-1),(i+1, s)\}$

as Figure 12(c) illustrates. First observe that $C$ must be double labeled by some number $c$, since otherwise none of the right most horizontal domino cells above $C$ can be double labeled and this contradicts to the fact that $T$ is a standard $r$-domino tableau.

(a)

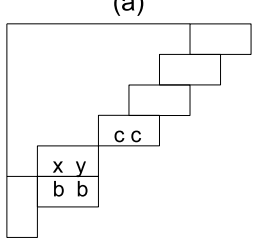

$\left(a^{\prime}\right)$

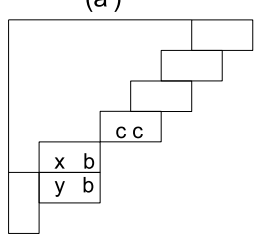

(b)

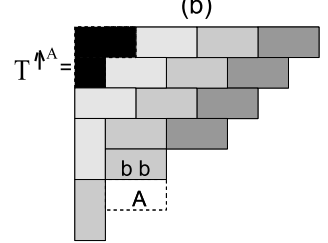

(c)

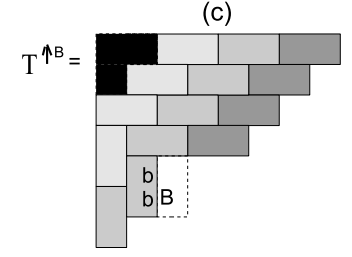

Figure 13: Case 3.2.2, continuation to Figure 12 (c)

Now we first suppose that $A$ is double labeled by some number $b$ and consider the horizontal domino cell $D=\{(i+2, s-3),(i+2, s-2)\}$ which is indicated by the labeling $(x, y)$ in Figure 13(a). Observe from Corollary 2.5 that Garfinkle's reverse insertion algorithm applied on $A$ first pushes back $[D,(x, y)]$ and then labels $D$ by $(b, b)$ as Figure $13(b)$ suggests. On the other hand Garfinkle's reverse insertion algorithm applied on $B$ first pushes back $[D,(x, y)]$ and then labels the vertical domino cell $\{(i+2, s-3),(i+3, s-3)\}$ by $(b, b)$ as Figure 13(c) illustrates. Since the domino cell $D$ of $T$ is to be pushed back in the first step of both reverse insertion, we have

$$
\alpha_{n}=\eta\left(T^{\uparrow A}\right)=z=\eta\left(T^{\uparrow B}\right)=\beta_{n}
$$

and furthermore the resulting tableaux $T^{\uparrow A}$ and $T^{\uparrow B}$ just differ by their domino cells labeled by $(b, b)$. If $B$ is double labeled by some number $b$, then one can get the same result except that, this time the vertical domino cell $D^{\prime}=\{(i+2, s-3),(i+3, s-3)\}$ (as Figure 13( $\left.a^{\prime}\right)$ indicates by the labeling $(x, y)$ ) is to be pushed back in the first step of Garfinkle's reverse insertion applied on $A$ and $B$.

Now we consider $T^{\uparrow B}$. Observe that the east most horizontal and the south most vertical domino cells of $T^{\uparrow B}$ must be double labeled. We first apply the reverse insertion on the east most horizontal domino cells from the bottom to the top, starting from $C$, so that a sequence of positive increasing numbers is obtained. Next the reverse insertion applied on the south most vertical domino cells from right to left gives a sequence of increasing negative numbers. Continuing the reverse insertion of the east most horizontal 
domino cells and the south most vertical domino cells in the remaining tableaux one at a time, we end up by a staircase shape $(r, r-1, \ldots, 0)$ for some $r \geq 0$.

If the shape $(r, r-1, \ldots, 0)$ is obtained by reverse inserting the east most horizontal domino cells at the end, as Figure 13 and Figure 2(a) illustrate, then last sequence obtained in this manner must be also positive decreasing as the first sequence. Therefore for some $k \geq 1$ satisfying $s+i=r+2(k+1)$ we have the following word

$$
u=\overbrace{a_{1, r+1} \ldots a_{1,1} b_{1,1}}^{\mathfrak{c}_{1}} \ldots \overbrace{a_{k, r+k} \ldots a_{k, 1} b_{k, k} \ldots b_{k, 1}}^{\mathfrak{c}_{k}} \overbrace{a_{k+1, r+k} \ldots a_{k+1,1}}^{\mathfrak{C}_{k+1}}
$$

where in $\mathfrak{C}_{i}, a_{i, r+i} \ldots a_{1,1}$ represents positive decreasing sequence obtained by reverse inserting east most horizontal domino cells and $b_{i, i} \ldots b_{1,1}$ represent negative increasing sequence obtained by reverse inserting south most vertical domino cells and $\mathfrak{C}_{1}$ represent the last sequence. Therefore $P(u)=T^{\uparrow B}$ and the numbers $a_{i, j}$ and $b_{i, j}$ in $u$ satisfy the following conditions.

$$
\begin{aligned}
& a_{i, j}>0, b_{i, j}<0 \\
& a_{i, j-1}<a_{i, j}<a_{i+1, j} \text { and }\left|b_{i, j-1}\right|<\left|b_{i, j}\right|<\left|b_{i+1, j}\right| \\
& \left|b_{i, i}\right|<a_{i+1, r+i+1}<\left|b_{i+1, i+1}\right| \text { for all } i=1, \ldots, k-1 .
\end{aligned}
$$

Consider the word $u^{\prime}$ which is obtained by taking $\left|b_{k, k}\right|$ (Observe that $b_{k, k}=b$ in Figure 2) in front of $a_{k, r+k}$ in $u$.

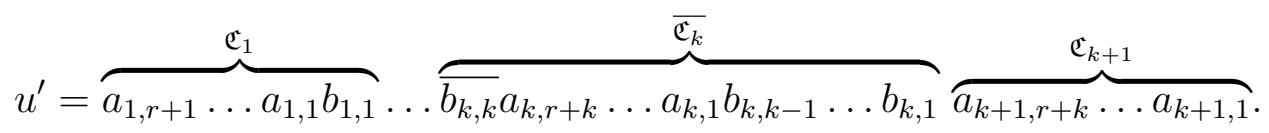

Therefore $P^{r}\left(u^{\prime}\right)=T^{\uparrow A}$ whereas $P^{r}(u)=T^{\uparrow B}$ and since $\left(T^{\uparrow B}\right)^{\downarrow z}=T=\left(T^{\uparrow A}\right)^{\downarrow z}$ we have

$$
P^{r}(u z)=T=P^{r}\left(u^{\prime} z\right) .
$$

Now we have the following analysis on $z=\eta\left(T^{\uparrow B}\right)=\eta\left(T^{\uparrow A}\right)$ : Observe that either $b$ or $c$ must be equal to $n$. If $b=n$ then any number $z$ between $b_{k, 1}$ and $a_{k+1,1}$ satisfies

$$
\left(T^{\uparrow B}\right)^{\downarrow z}=T=\left(T^{\uparrow A}\right)^{\downarrow z}
$$

where $\left|b_{k, 1}\right|$ and $a_{k+1,1}$ respectively are the labels of south most vertical and right most horizontal domino cells in both $T^{\uparrow B}$ and $T^{\uparrow A}$. If $c=n$ then insertion of the number $z$ in both $T^{\uparrow B}$ and $T^{\uparrow A}$ can not bump the domino cell $C$ which is double labeled by $n$ in to the next row since then resulting tableau is not equal to $T$. Therefore in this case

$$
\begin{aligned}
& \text { either } b_{k, 1}<z<a_{k, 1} \\
& \quad \text { or } a_{k, 1}<z<a_{k+1,1} \text { and } a_{k, i+1}<a_{k+1, i} \text { for some } 1<i \leq k-1 .
\end{aligned}
$$

As a result two words $u z$ and $u^{\prime} z$ satisfy $D_{4}^{r}$ relation with all $a_{i, j}>0$ and $b_{i, j}<0$ and so $u z \stackrel{p_{r}}{\sim} u^{\prime} z$ 
Now if the shape $(r, r-1, \ldots, 0)$ is obtained by reverse inserting the south most vertical domino cells at the end (see for example Figure 2(b)) then last sequence obtained in this manner must be negative increasing as opposed to the first sequence. Therefore for some $k \geq 1$ satisfying $s+i=r+2(k+1)+1$, we have the following word

$$
u=\overbrace{a_{1, r+1} \ldots a_{1,1} b_{1,1}}^{\mathfrak{c}_{1}} \ldots \overbrace{a_{k, r+k} \ldots a_{k, 1} b_{k, k} \ldots b_{k, 1}}^{\mathfrak{c}_{k}} \overbrace{a_{k+1, r+k+1} \ldots a_{k+1,1} b_{k+1, k} \ldots b_{k+1,1}}^{\mathfrak{C}_{k+1}}
$$

where in $\mathfrak{C}_{i}, a_{i, r+i} \ldots a_{1,1}$ represent negative increasing sequence obtained by reverse inserting south most vertical domino cells and $b_{i, i} \ldots b_{1,1}$ represent positive decreasing sequence obtained by reverse inserting east most horizontal domino cells and $\mathfrak{C}_{1}$ represent the last sequence. Moreover the numbers $a_{i, j}$ and $b_{i, j}$ in $u$ satisfy the following conditions.

$$
\begin{aligned}
& a_{i, j}<0 \text { and } b_{i, j}>0 \\
& \left|a_{i, j-1}\right|<\left|a_{i, j}\right|<\left|a_{i+1, j}\right| \text { and } b_{i, j-1}<b_{i, j}<b_{i+1, j} \\
& \left|a_{i, r+i}\right|<b_{i, i}<\left|a_{i+1, r+i+1}\right| \text { for all } i=1, \ldots, k
\end{aligned}
$$

Now one can easily check the following word

$$
u^{\prime}=\overbrace{a_{1, r+1} \ldots a_{1,1} b_{1,1}}^{\mathfrak{C}_{1}} \ldots \overbrace{a_{k, r+k} \ldots a_{k, 1} \overline{a_{k+1, r+k+1}} b_{k, k} \ldots b_{k, 1}}^{\overline{\mathfrak{c}_{k}}} \overbrace{a_{k+1, r+k} \ldots a_{k+1,1} b_{k+1, k} \ldots b_{k+1,1}}^{\overline{\mathfrak{c}_{k+1}}}
$$

satisfies $P^{r}\left(u^{\prime}\right)=T^{\uparrow A}$ whereas $P^{r}(u)=T^{\uparrow B}$. Moreover a similar analysis on the number $z$ shows that $z$ satisfies one of the hypothesis of $D_{5}^{r}$, therefore two words $u z$ and $u^{\prime} z$ satisfy $D_{5}^{r}$ relation with all $a_{i, j}<0$ and $b_{i, j}>0$ and so $u z \stackrel{p_{r}}{\sim} u^{\prime} z$.

Note that for the tableaux that Figure 2(c) and (d) illustrates, one needs to apply the reverse insertion the south most vertical domino cells from left to right starting from $C$ and in that case a sequence of negative decreasing numbers is obtained. Moreover, according to the sign of the last sequence obtained in the same manner one get either $D_{4}^{r}$ relation with all $a_{i, j}<0$ and $b_{i, j}>0$ or $D_{5}^{r}$ relation with all $a_{i, j}>0$ and $b_{i, j}<0$ as Figure 2(c)and Figure 2(d) illustrates respectively.

Now recall that $P(u)=T^{\uparrow A}=P\left(\alpha_{1} \ldots \alpha_{n-1}\right)$ and $P\left(u^{\prime}\right)=T^{\uparrow B}=P\left(\beta_{1} \ldots \beta_{n-1}\right)$. So we have

$$
\alpha_{n}=z=\beta_{n}
$$

and also $u \stackrel{p_{r}}{\sim} \alpha_{1} \ldots \alpha_{n-1}$ and $u^{\prime} \stackrel{p_{r}}{\sim} \beta_{1} \ldots \beta_{n-1}$ by induction. Therefore $\alpha \stackrel{p_{r}}{\sim} u z \stackrel{p_{r}}{\sim} u^{\prime} z \stackrel{p_{r}}{\sim} \beta$ as desired.

Case 4: $A \neq B,(T, A$, ne $) \cap(T, B, \mathrm{sw})$ is a staircase shape $(s, s-1, \ldots, 1)$ for $s \geq 1$ and $A \cap B$ is empty. Figure 14 shows several subcases.

Case 4.1: We first assume that there is another domino corner $C$ of $T$. Without loss of generality we assume that $C$ lies in $(T, B$, ne) since the other case can be dealt with in the same manner after taking the transpose of $T$.

Case 4.1.1: Suppose that $(\mathrm{T}, \mathrm{C}, \mathrm{sw}) \cap(T, B, \mathrm{ne})$ contains a domino corner as in Figure 14(a). Let $\sigma$ be a permutation in $B_{n}$ such that $T^{\uparrow C}=P^{r}\left(\sigma_{1} \ldots \sigma_{n-1}\right)$ and $\eta\left(T^{\uparrow C}\right)=\sigma_{n}$. 
(a)

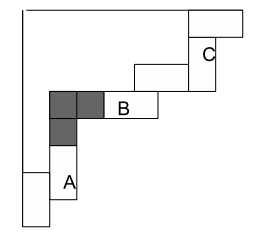

(b)

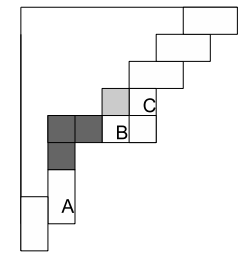

Figure 14: Case 4. (c)

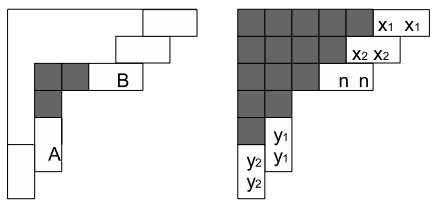

Then the pairs of domino corners $A$ and $C$ and similarly $B$ and $C$ satisfy Case 2 . Therefore we have $\beta \stackrel{p_{r}}{\sim} \sigma \stackrel{p_{r}}{\sim} \alpha$. The case when $C$ lies in $(T, A, \mathrm{sw})$ follows similarly.

Case 4.1.2: Now suppose that $(\mathrm{T}, \mathrm{C}, \mathrm{sw}) \cap(T, B$, ne) is a staircase shape $(s, s-1, \ldots, 2,1)$ for some $s \geq 1$. Observe that the case $s>1$ is impossible since $B$ is a horizontal domino cell. For $s=1$ consider Figure 14(b). Let $\sigma$ be a permutation in $B_{n}$ such that $T^{\uparrow C}=P^{r}\left(\sigma_{1} \ldots \sigma_{n-1}\right)$ and $\eta\left(T^{\uparrow C}\right)=\sigma_{n}$. Then as the domino corners $B$ and $C$ satisfy Case 3.1.1, we have $\beta \stackrel{p_{r}}{\sim} \sigma$. On the other hand $(T, A$, ne $) \cap(T, C, \mathrm{sw})$ also contains a domino corner, therefore by Case 2 we have $\alpha \stackrel{p_{r}}{\sim} \sigma$. Hence $\alpha \stackrel{p_{r}}{\sim} \beta$. The case when $C$ lies in $(T, A, \mathrm{sw})$ also follows similarly.

Case 4.2: Now we suppose that there is no domino corner of $T$ beyond $A$ and $B$ as illustrated in Figure $14(c)$. One can easily see that after reverse insertion all horizontal domino cells and then vertical domino cells, only a staircase shape is left. Therefore $n \leq r+1$ and $\eta\left(T^{\uparrow A}\right)=\alpha_{n}<0$ and $\eta\left(T^{\uparrow B}\right)=\beta_{n}>0$. Moreover we have

$$
T^{\uparrow A \uparrow B}=T^{\uparrow B \uparrow A}, \eta\left(T^{\uparrow A \uparrow B}\right)=\beta_{n} \text { and } \eta\left(T^{\uparrow B \uparrow A}\right)=\alpha_{n} .
$$

Let $u$ be a signed word such that $P^{r}(u)=T^{\uparrow A \uparrow B}=T^{\uparrow B \uparrow A}$. Clearly $P^{r}\left(u \alpha_{n} \beta_{n}\right)=T=$ $P^{r}\left(u \beta_{n} \alpha_{n}\right)$ and the size of $u$ is less than $r-1$. Moreover

$$
u \alpha_{n} \beta_{n} \stackrel{\mathrm{D}_{2}^{r}}{\sim} u \beta_{n} \alpha_{n}
$$

On the other hand $P^{r}\left(u \beta_{n}\right)=T^{\uparrow A}$ and $P^{r}\left(u \alpha_{n}\right)=T^{\uparrow B}$ and by induction we have $\alpha_{1} \ldots \alpha_{n-1} \stackrel{p_{r}}{\sim} u \beta_{n}$ and $\beta_{1} \ldots \beta_{n-1} \stackrel{p_{r}}{\sim} u \alpha_{n}$. Hence as desired

$$
\alpha=\alpha_{1} \ldots \alpha_{n-1} \alpha_{n} \stackrel{p_{r}}{\sim} u \beta_{n} \alpha_{n} \stackrel{\mathrm{D}_{2}^{r}}{\sim} u \alpha_{n} \beta_{n} \stackrel{p_{r}}{\sim} \beta_{1} \ldots \beta_{n-1} \beta_{n}=\beta
$$

\section{References}

[1] D. Barbash, D. Vogan, Primitive ideals and orbital integrals in complex classical groups, Math. Ann., 259, (1982), 153-199.

[2] C. Bonnafé, On Kazhdan-Lusztig cells in type B, J. Alg. Combin. 31 (2010), 53-82.

[3] C. Bonnafé, M. Geck, L. Iancu, T.Lam, On the insertion and Kazhdan-Lusztig cells in type $B_{n}$, math.RT/0609279. Progress in Math (Lusztig Birthday Volume), Birkhauser, to appear. 
[4] D. Garfinkle, On the classification of primitive ideals for complex classical Lie algebras I, Compositio Mathematica 75 (1990), 135-169.

[5] D. Garfinkle, On the classification of primitive ideals for complex classical Lie algebras II, Compositio Mathematica 81 (1992), 307-336.

[6] D. Garfinkle, On the classification of primitive ideals for complex classical Lie algebras III, Compositio Mathematica 88 (1993), 187-234.

[7] M. Geck, Left cells and constructible representations, Representation Theory 9, (2005), 385-416.

[8] I. G. Gordon, Quiver varieties, category $\mathcal{O}$ for rational Cherednik algebras, and Hecke algebras, Int. Math. Res. Pap. IMRP, 3 (2008), 69 p.

[9] I.G. Gordon, M. Martino, Calogero-Moser spaces, restricted rational Cherednik algebras, and two-sided cells, Math. Res. Lett., 16 (2009), 255-262.

[10] A. Joseph, On the variety of a highest weight module, J. Algebra, 88, (1984), 238-278.

[11] D. Kazhdan, G. Lusztig, Representations of Coxeter groups and Hecke algebras, Invent. Math., 53 (1979), 165-184.

[12] D. E. Knuth, Permutations, matrices and generalized Young tableaux, Pacific J. Math., 34 (1970), 709-727.

[13] M.A.A. van Leeuwen, The Robinson-Schensted and Schützenberger algorithms, an elementary approach, Electron. J. Combin. 3 (1996).

[14] G. Lusztig, Hecke algebras with unequal parameters, CRM Monographs Ser.18, Amer. Math. Soc. (2003), 136p.

[15] W. McGovern, Left cells and domino tableaux in classical Weyl groups, Composito Mathematica 101 (1996), 77-98.

[16] T. Pietraho, Cells in the Weyl Groups of type B(n), J. Alg. Combin. 27(2) (2008), $247-262$.

[17] T. Pietraho, Cells and Constructible Representations in Type B, New York J. Math. 14 (2008), 411-430.

[18] T. Pietraho, Knuth Relations for the Hyperoctahedral Groups, J. Alg. Combin. 29, (2009) 509-535.

[19] C. Schensted, Longest increasing and decreasing subsequences, Canad. J. Math. 13, (1961), 179-191.

[20] M. P. Schützenberger, Quelques remarques sur une construction de Schensted, Math. Scand. 12 (1963), 117-128.

[21] M. P. Schützenberger, La correspondence de Robinson, Combinatoire et Représentation du Groupe Symétrique, Lecture Notes in Math. 579, Springer, Berlin (1977), 59-135.

[22] M. Shimozono, D. White, A color-to-spin domino Schensted algorithm. Electronic J. Combin. 8, no. 1, (2001), Research Paper 21, 50 pp.

[23] D. Vogan, Ordering of the primitive spectrum of a semisimple Lie algebra, Math. Ann. 248 (1980), 195-2003. 